\title{
On the Drinfeld moduli problem of $p$-divisible groups
}

\author{
Michael Rapoport* and Thomas ZinK ${ }^{\dagger}$
}

\begin{abstract}
Drinfeld proved that the $p$-adic upper half space $\Omega_{F}^{d}$ for a $p$-adic local field $F$ is the general fiber of a formal scheme which is the moduli space of special formal $O_{D}$-modules, where $D$ is a central division algebra with invariant $1 / d$ over $F$. We give examples of other moduli problems of $p$-divisible formal groups which have general fiber $\Omega_{F}^{d}$. We show that, when $F / \mathbb{Q}_{p}$ is unramified, our moduli spaces actually agree with Drinfeld's space. Using our results in the ramified case, P. Scholze has proved this in general. We also consider a variant for the Lubin-Tate moduli problem.
\end{abstract}

\section{Introduction}

2 Formulation of the main results

3 The Kottwitz and Eisenstein conditions

4 Formal $O_{D}$-modules

5 The local model

6 The generic fiber (after Scholze)

7 The unramified case

8 The Lubin-Tate moduli problem

\section{References}

*Research of Rapoport supported by SFB/TR 45 "Periods, Moduli Spaces and Arithmetic of Algebraic Varieties" of the DFG.

${ }^{\dagger}$ Research of Zink supported by SFB 701 "Spectral Structures and Topological Methods in Mathematics" of the DFG. 


\section{Introduction}

Let $F$ be a finite extension of $\mathbb{Q}_{p}$, with ring of integers $O_{F}$ and uniformizer $\pi$. In [5], Drinfeld defines a certain moduli problem of formal $O_{F}$-modules. Let us recall Drinfeld's theorem.

A formal $O_{F}$-module over a scheme $S$ such that $p$ is locally nilpotent in $\mathcal{O}_{S}$ is a $p$-divisible formal group $X$ over $S$ with an action of $O_{F}$,

$$
\iota: O_{F} \longrightarrow \text { End } X \text {. }
$$

If $X$ is defined over an $O_{F}$-scheme $S$, the Lie algebra Lie $X$ is naturally an $O_{F}$-module. If this coincides with the $O_{F}$-module structure given by $d \iota$ we call $X$ a strict formal $O_{F}$-module.

Let $D$ be a central division algebra of invariant $1 / n$ over $F$, with maximal order $O_{D}$. Drinfeld defines a special formal $O_{D}$-module over an $O_{F}$-scheme $S$ to be a strict formal $O_{F}$-module of height $n^{2}\left[F: \mathbb{Q}_{p}\right]$ equipped with an action $\iota: O_{D} \rightarrow \operatorname{End}(X)$ of $O_{D}$ that extends the action of $O_{F}$ and such that, in each geometric point of $S$, the eigenspaces of Lie $X$ under the action of an unramified extension $O_{\tilde{F}}$ of $O_{F}$ of degree $n$ contained in $O_{D}$ are all one-dimensional.

It is easy to see that if $S=\operatorname{Spec} \bar{k}$ is the spectrum of the algebraic closure of the residue field of $O_{F}$, there is a unique special formal $O_{D}$-module $\mathbb{X}$ over $S$, up to $O_{D}$-linear isogeny.

Let $O_{\breve{F}}$ be the ring of integers in the completion of the maximal unramified extension $\breve{F}$ of $F$. Drinfeld defines as follows a set-valued functor $\mathcal{M}$ on the category $\operatorname{Nilp}_{O_{\breve{F}}}$ of $O_{\breve{F}}$-schemes $S$ such that the ideal sheaf $\pi \mathcal{O}_{S}$ is locally nilpotent: the functor associates to $S \in$ Nilp $_{O_{\breve{F}}}$ the set of isomorphism classes of triples $(X, \iota, \rho)$, where $(X, \iota)$ is a special formal $O_{D}$-module over $S$ and where $\rho: X \times_{S} \bar{S} \rightarrow \mathbb{X} \times_{\text {Spec } \bar{k}} \bar{S}$ is a $O_{D}$-linear quasi-isogeny of height 0 . Here $\bar{S}=S \times{ }_{\operatorname{Spec}} O_{\breve{F}} \operatorname{Spec} \bar{k}$. Drinfeld's theorem is that this functor is representable by a very specific formal scheme, namely,

$$
\mathcal{M} \simeq \hat{\Omega}_{F}^{n} \times_{\operatorname{Spf} O_{F}} \operatorname{Spf} O_{\breve{F}} .
$$

Here $\hat{\Omega}_{F}^{n}$ is the formal scheme over $\operatorname{Spf} O_{F}$ defined by Deligne, Drinfeld and Mumford, cf. [5]. It has as generic fiber (associated rigid-analytic space) Drinfeld's $p$-adic halfspace associated to $F$,

$$
\left(\hat{\Omega}_{F}^{n}\right)^{\mathrm{rig}}=\mathbb{P}_{F}^{n-1} \backslash \bigcup_{H / F} H .
$$

Here $H$ ranges over the hyperplanes of $\mathbb{P}_{F}^{n-1}$ defined over $F$. 
Drinfeld's theorem has many applications, in particular to the $p$-adic uniformization of Shimura varieties, cf. [21]. It also has applications to arithmetic, e.g. [16, 23].

The generic fiber of Drinfeld's formal moduli space admits a tower of finite étale coverings (via level structures on the Tate module of the universal $p$-divisible group). As such it is a prominent example of a local Shimura variety, cf. [20].

Let $G$ be a reductive group over the local field $F$, let $b$ be an element of $G(\breve{F})$ and let $\{\mu\}$ be a conjugacy class of minuscule cocharacters of $G_{\bar{F}}$. One requires that the $\sigma$-conjugacy class $[b]$ of $b$ is neutral acceptable, i.e., $[b] \in B(G,\{\mu\})$, cf. [10]. The triple $(G, b,\{\mu\})$ is called a local Shimura datum over $F$, cf. [20]. One expects to be able to attach to these data a local Shimura variety which satisfies obvious functorial properties and more. This is a tower of rigid-analytic spaces $\mathbb{M}(G, b,\{\mu\})=\left\{\mathbb{M}^{K} \mid K \subset G(F)\right\}$ indexed by the open compact subgroups of $G(F)$, defined over the reflex field $E=E(G,\{\mu\})$, cf. [20]. By [21], local Shimura varieties exist in many cases. In the PEL case, local Shimura varieties are related to Shimura varieties by non-archimedean uniformization, cf. [21, Thm. 6.36]. One also expects to have integral models over $O_{E}$ of $\mathbb{M}^{K}$, for judicious choices of the open compact subgroup $K$, i.e., formal schemes over $\operatorname{Spf} O_{\breve{E}}$ with Weil descent datum to $\operatorname{Spf} O_{E}$ whose associated rigid-analytic space is $\mathbb{M}^{K}$.

In Drinfeld's case $G=D^{\times}$, (the linear algebraic group over $F$ associated to) the multiplicative group of $D$. The cocharacter $\{\mu\}$ of $G_{\bar{F}} \simeq \mathrm{GL}_{n, \bar{F}}$ is $(1,0, \ldots, 0)$ and $b$ is a representative of the unique element of $B(G,\{\mu\})$. Drinfeld's theorem says not only that the member $\mathbb{M}^{K}$, for the level subgroup $K=O_{D}^{\times}$, is the Drinfeld $p$-adic upper half space attached to $F$, but also that the moduli problem $\mathcal{M}$ defines an integral model of $\mathbb{M}^{K}$, which is even a $\pi$-adic formal scheme with semi-stable reduction.

In the theory of local Shimura varieties, the following question arises. Assume that $b$ is a representative of the unique basic element of $B(G,\{\mu\})$. Let $\varepsilon$ be a central cocharacter of $G$ defined over $F$, and set $\left\{\mu^{\prime}\right\}=\{\mu \varepsilon\}$. Then $E(G,\{\mu\})=E\left(G,\left\{\mu^{\prime}\right\}\right)$. Let $b^{\prime}$ be a representative of the unique basic element of $B\left(G,\left\{\mu^{\prime}\right\}\right)$. The question is whether the local Shimura varieties $\mathbb{M}(G, b,\{\mu\})$ and $\mathbb{M}\left(G, b^{\prime},\left\{\mu^{\prime}\right\}\right)$ are Galois twists of each other (unramified twists if the connected center of $G$ splits over an unramified extension).

In a similar vein, start with a local Shimura datum $(G, b,\{\mu\})$ over $F$ such that $b$ is basic. Let $G^{\prime}=\operatorname{Res}_{F / \mathbb{Q}_{p}}(G)$. Then $G^{\prime} \otimes_{\mathbb{Q}_{p}} \overline{\mathbb{Q}}_{p}$ is a product of $G \otimes_{F} \overline{\mathbb{Q}}_{p}$ indexed by the embeddings of $F$ in $\overline{\mathbb{Q}}_{p}$. We fix an embedding by choosing an isomorphism $\bar{F} \cong \overline{\mathbb{Q}}_{p}$. Define the conjugacy class $\left\{\mu_{0}\right\}$ of $G^{\prime}$ to be $\{\mu\}$ for this fixed embedding and to be trivial for all other embeddings. Then 
$E\left(G^{\prime},\left\{\mu_{0}\right\}\right) \subset E(G,\{\mu\})$ with respect to the chosen isomorphism. Let $\varepsilon$ be a central cocharacter of $G^{\prime}$ defined over $F \subset \overline{\mathbb{Q}}_{p}$. We set $\left\{\mu^{\prime}\right\}=\left\{\mu_{0} \varepsilon\right\}$. We still have $E\left(G^{\prime},\left\{\mu^{\prime}\right\}\right) \subset E(G,\{\mu\})$. Let $b^{\prime}$ be a representative of the unique basic element in $B\left(G^{\prime},\left\{\mu^{\prime}\right\}\right)$. The question is whether the local Shimura varieties $\mathbb{M}(G, b,\{\mu\})$ and $\mathbb{M}\left(G^{\prime}, b^{\prime},\left\{\mu^{\prime}\right\}\right) \otimes_{E\left(G^{\prime},\left\{\mu^{\prime}\right\}\right)} E(G,\{\mu\})$ are Galois twists of each other (unramified twists if the connected center of $G^{\prime}$ splits over an unramified extension of $F$ ).

The goal of this paper is to prove that this last question has an affirmative answer for the Drinfeld datum $(G, b,\{\mu\})$. But, even better, we construct integral models for the members corresponding to the natural maximal level subgroups of both local Shimura varieties and show that they are isomorphic, at least when $F / \mathbb{Q}_{p}$ is unramified ${ }^{1}$. These integral models are constructed by posing a moduli problem of $p$-divisible groups. This is substantially different from Drinfeld's moduli problem (unless $F=\mathbb{Q}_{p}$ ), which is a moduli problem of strict formal $O_{F}$-modules. This contrast between relative and absolute Rapoport-Zink spaces is important also in other contexts: in the work of A. Mihatsch on the Arithmetic Fundamental Lemma [15] and in joint work of us with S. Kudla [13] on p-adic uniformization of Shimura curves. In fact, the approach in [13] is modelled on the present paper, but involves in addition a polarization.

There is another well-known local Shimura datum $(G, b,\{\mu\})$, referred to as the Lubin-Tate datum. Here $G=\mathrm{GL}_{n},\{\mu\}=(1,0, \ldots, 0)$, and $[b] \in$ $B(G,\{\mu\})$ is the unique basic element. In this case, the corresponding local Shimura variety again has an explicit integral model for its member $\mathbb{M}^{K}$, where $K=\mathrm{GL}_{n}\left(O_{F}\right)$. For this local Shimura variety, we also give a positive answer to the question raised above, again in the strong form pertaining to integral models. This theorem is applied in the work of B. Smithling, W. Zhang and the second author on the Arithmetic Gan-Gross-Prasad conjecture [22].

It should be pointed out that the two cases of integral models of $\mathbb{M}^{K}$ considered here are essentially the only ones known explicitly, which justifies singling out these special cases of a general problem.

The lay-out of the paper is as follows. In section 2 we formulate our moduli functor and state our main results. In section 3, we discuss the conditions on the Lie algebras in the formulation of the moduli problem. In section 4 we establish an isomorphism between our moduli functor when

\footnotetext{
${ }^{1}$ In his talk 14 July 2016 in the Bonn Arbeitsgemeinschaft Arithmetische Geometrie, P. Scholze explained his proof of Conjecture 2.6 below, i.e., how to remove the unramifiedness hypothesis. His proof is based on our Theorem 2.8, but uses in addition the integral p-adic Hodge theory of B. Bhatt, M. Morrow and P. Scholze (arXiv:1602.03148), and more. Scholze will publish his proof elsewhere.
} 
restricted to $\bar{k}$-schemes with the original Drinfeld moduli functor. The main tool here is the theory of displays. In section 5 we determine the local structure of our moduli scheme. Here the main tool is the theory of local models of Rapoport-Zink spaces. In section 6 we prove the compatibility theorem with the Drinfeld moduli functor in the generic fiber. This proof is due to P. Scholze, and uses his theory of $p$-divisible groups over $O_{C}$, cf. [24]. In section 7 we prove our integral representability conjecture in the case where $F / \mathbb{Q}_{p}$ is unramified. The proof uses the theory of relative displays of T. Ahsendorf. In the final section 8 we give the Lubin-Tate variant of our main theorem.

\section{Formulation of the main results}

Let $F$ be a field extension of degree $d$ of $\mathbb{Q}_{p}$. We denote by $O_{F}$ the ring of integers and by $\kappa$ the residue field. We write $f=\left[\kappa: \mathbb{F}_{p}\right]$ for the inertia index and $d=e f$.

Fix $n \geq 2$. Let $\Phi=\operatorname{Hom}_{\mathbb{Q}_{p}}\left(F, \overline{\mathbb{Q}}_{p}\right)$ be the set of field embeddings. We fix an embedding $\varphi_{0}: F \rightarrow \overline{\mathbb{Q}}_{p}$. Let $r: \Phi \rightarrow \mathbb{Z}$ be a function such that

$$
r_{\varphi}= \begin{cases}1, & \text { if } \varphi=\varphi_{0} \\ 0 \text { or } n, & \text { if } \varphi \neq \varphi_{0} .\end{cases}
$$

The reflex field $E \subset \overline{\mathbb{Q}}_{p}$ of $r$ is characterized by

$$
\operatorname{Gal}\left(\overline{\mathbb{Q}}_{p} / E\right)=\left\{\sigma \in \operatorname{Gal}\left(\overline{\mathbb{Q}}_{p} / \mathbb{Q}_{p}\right) \mid r_{\sigma \varphi}=r_{\varphi} \text { for all } \varphi\right\} .
$$

We have $\varphi_{0}(F) \subset E$, and we consider $E$ as a field extension of $F$ via $\varphi_{0}$.

Let $D$ be a central division algebra of invariant $1 / n$ over $F$. We will consider $p$-divisible groups $X$ of height $n^{2} d$ over $O_{E}$-schemes $S$, with an action of the ring of integers $O_{D}$ in $D$,

$$
\iota: O_{D} \longrightarrow \operatorname{End}(X) \text {. }
$$

We will need to impose conditions on the induced action of $O_{D}$ on Lie $X$. The first condition is the Kottwitz condition

$$
\operatorname{char}(\iota(x) \mid \operatorname{Lie} X)=\prod_{\varphi} \varphi(\operatorname{chard}(x)(T))^{r_{\varphi}}, \quad \forall x \in O_{D} .
$$

Here chard $(x)$ denotes the reduced characteristic polynomial of $x$, a polynomial of degree $n$ with coefficients in $O_{F}$. The RHS is a polynomial in $O_{E}[T]$. It becomes a polynomial with coefficients in $\mathcal{O}_{S}$ via the structure morphism. 
As we will show (cf. Proposition 2.2), the Kottwitz condition is all we need to yield a good moduli problem when $F / \mathbb{Q}_{p}$ is unramified. When $F / \mathbb{Q}_{p}$ is ramified, the Kottwitz condition is too weak. To state the additional condition we impose, we need some preparation.

Let $F^{t}$ be the maximal unramified subfield of $F$. We will write $\Psi=$ $\operatorname{Hom}_{\mathbb{Q}_{p}}\left(F^{t}, \overline{\mathbb{Q}}_{p}\right)$ for the set of field embeddings. Let $\psi_{0}=\varphi_{0 \mid F^{t}}$. For an embedding $\psi: F^{t} \rightarrow \overline{\mathbb{Q}}_{p}$ we set

$$
\begin{aligned}
& A_{\psi}=\left\{\varphi: F \rightarrow \overline{\mathbb{Q}}_{p} \mid \varphi_{\mid F^{t}}=\psi, \text { and } r_{\varphi}=n\right\} \\
& B_{\psi}=\left\{\varphi: F \rightarrow \overline{\mathbb{Q}}_{p} \mid \varphi_{\mid F^{t}}=\psi, \text { and } r_{\varphi}=0\right\} .
\end{aligned}
$$

Also, let $a_{\psi}=\left|A_{\psi}\right|$ and $b_{\psi}=\left|B_{\psi}\right|$. For any $O_{E}$-scheme $S$ we have a decomposition of $O_{F^{t}} \otimes_{\mathbb{Z}_{p}} \mathcal{O}_{S^{-} \text {-modules }}$

$$
O_{F^{t}} \otimes_{\mathbb{Z}_{p}} \mathcal{O}_{S}=\bigoplus_{\psi \in \Psi} \mathcal{O}_{S}
$$

where the action of $\mathcal{O}_{F^{t}}$ on the $\psi$-th factor is via $\psi$. Hence for $(X, \iota)$ over $S$, we obtain a decomposition into locally free $\mathcal{O}_{S}$-modules,

$$
\text { Lie } X=\bigoplus_{\psi \in \Psi} \operatorname{Lie}_{\psi} X
$$

The rank of $\operatorname{Lie}_{\psi} X$ is given by (2.2) as

$$
\operatorname{rank} \operatorname{Lie}_{\psi} X=a_{\psi} n^{2}+\epsilon_{\psi} n
$$

where $\epsilon_{\psi}$ is equal to 1 if $\psi=\psi_{0}$, and is equal to 0 if $\psi \neq \psi_{0}$.

Let $\pi$ be a uniformizer in $O_{F}$. Consider the Eisenstein polynomial $Q(T)$ of $\pi$ in $O_{F^{t}}[T]$. We consider the image $Q_{\psi}(T)$ of $Q(T)$ in $\overline{\mathbb{Q}}_{p}[T]$ under $\psi$, for $\psi \in \Psi$. In $\overline{\mathbb{Q}}_{p}[T]$ this has a decomposition into linear factors,

$$
Q_{\psi}(T)=\prod_{\left\{\varphi \mid \varphi_{\left.\mid F^{t}=\psi\right\}}\right.}(T-\varphi(\pi))
$$

Note that $\operatorname{Gal}\left(\overline{\mathbb{Q}}_{p} / E\right)$ acts on the index set of this product, as is clear since the LHS is a polynomial in $O_{E}[T]$. We therefore obtain a decomposition in $O_{E}[T]$

$$
\begin{aligned}
Q_{\psi_{0}}(T) & =Q_{0}(T) \cdot Q_{A_{\psi_{0}}}(T) \cdot Q_{B_{\psi_{0}}}(T), & \operatorname{resp.} \\
Q_{\psi}(T) & =Q_{A_{\psi}}(T) \cdot Q_{B_{\psi}}(T), & \text { for } \psi \neq \psi_{0} .
\end{aligned}
$$


Here

$Q_{0}(T)=T-\varphi_{0}(\pi), Q_{A_{\psi}}(T)=\prod_{\varphi \in A_{\psi}}(T-\varphi(\pi)), Q_{B_{\psi}}(T)=\prod_{\varphi \in B_{\psi}}(T-\varphi(\pi))$.

Indeed, the action of $\operatorname{Gal}\left(\overline{\mathbb{Q}}_{p} / E\right)$ stabilizes the corresponding subsets in the index set on the RHS of (2.7). Using the structure morphism $O_{E} \rightarrow \mathcal{O}_{S}$, we obtain an endomorphism $Q_{A_{\psi}}(\iota(\pi))$ of the $\mathcal{O}_{S}$-module $\operatorname{Lie}_{\psi} X$ that we denote by $Q_{A_{\psi}}\left(\iota(\pi) \mid \operatorname{Lie}_{\psi} X\right)$. We similarly define $Q_{B_{\psi}}\left(\iota(\pi) \mid \operatorname{Lie}_{\psi} X\right)$ and $Q_{0}\left(\iota(\pi) \mid \operatorname{Lie}_{\psi_{0}} X\right)=\iota(\pi) \mid \operatorname{Lie}_{\psi_{0}} X-\varphi_{0}(\pi) \operatorname{Id}_{\operatorname{Lie}_{\psi_{0}} X}$.

The additional conditions we impose, that we call the Eisenstein conditions, are now the following identities of endomorphisms

$$
\begin{aligned}
\left(Q_{0} \cdot Q_{A_{\psi_{0}}}\right)\left(\iota(\pi) \mid \operatorname{Lie}_{\psi_{0}} X\right) & =0, \\
\bigwedge^{n+1}\left(Q_{A_{\psi_{0}}}\left(\iota(\pi) \mid \operatorname{Lie}_{\psi_{0}} X\right)\right) & =0, \\
Q_{A_{\psi}}\left(\iota(\pi) \mid \operatorname{Lie}_{\psi} X\right) & =0, \forall \psi \neq \psi_{0} .
\end{aligned}
$$

Remark 2.1. We note that the Eisenstein conditions only depend on the restriction of the $O_{D}$-action to $O_{F}$. It will follow a posteriori from Corollary 5.9 (flatness) that the moduli problem formulated using the Eisenstein conditions is independent of the choice of the uniformizer $\pi$.

We first note the following statement.

Proposition 2.2. If $F / \mathbb{Q}_{p}$ is unramified, the Eisenstein conditions are implied by the Kottwitz condition.

Proof. When $F=F^{t}$ is unramified over $\mathbb{Q}_{p}$, the uniformizer $\pi$ lies in $F^{t}$ and $Q(T)=T-\pi$ is a linear polynomial. Furthermore, $A_{\psi}$ has at most one element for $\psi \neq \psi_{0}$, and $A_{\psi_{0}}=\emptyset$. Let $\psi \neq \psi_{0}$. If $A_{\psi}=\emptyset$, then $\operatorname{Lie}_{\psi} X=(0)$ and the Eisenstein condition relative to the index $\psi$ is empty; if $A_{\psi}$ has one element, the Eisenstein condition relative to the index $\psi$ is just equivalent to the definition of the $\psi$-th eigenspace in the decomposition (2.5). Something analogous applies to the index $\psi_{0}$.

The following statement shows that the moduli problems considered in this paper are indeed generalizations of Drinfeld's moduli problem. We call the Drinfeld function the function $r^{\circ}$ with $r_{\varphi}^{\circ}=0, \forall \varphi \neq \varphi_{0}$. In this case $E_{r^{\circ}}=F$.

Proposition 2.3. Assume that $r=r^{\circ}$. Then a p-divisible group $(X, \iota)$ as above, i.e., satisfying the Kottwitz condition (2.2) and the Eisenstein conditions (2.9), is a special formal $O_{D}$-module in the sense of Drinfeld [5]. 
Proof. In this case, $A_{\psi}=\emptyset, \forall \psi$. Hence $\operatorname{Lie}_{\psi} X=(0)$ for $\psi \neq \psi_{0}$, and Lie $_{\psi_{0}} X$ is a locally free $\mathcal{O}_{S}$-module of rank $n$. Also, the endomorphism $Q_{A_{\psi_{0}}}\left(\iota(\pi) \mid \operatorname{Lie}_{\psi_{0}} X\right)$ is the identity automorphism. Hence the first Eisenstein condition implies that $Q_{0}\left(\iota(\pi) \mid \operatorname{Lie}_{\psi_{0}} X\right)=0$. Since $Q_{0}(T)=T-\varphi_{0}(\pi)$, it follows that $\iota(\pi)$ acts on $\operatorname{Lie}_{\psi_{0}} X$ through the structure morphism $O_{E} \rightarrow \mathcal{O}_{S}$. The same is true for all elements of $O_{F^{t}}$. Hence $X$ is a strict formal $O_{F^{-}}$ module. Now the Kottwitz condition (2.2) tells us that the action of $O_{D}$ on $X$ is special, which proves the claim.

Definition 2.4. Fix a function $r: \Phi \rightarrow \mathbb{Z}_{\geq 0}$, with corresponding reflex field

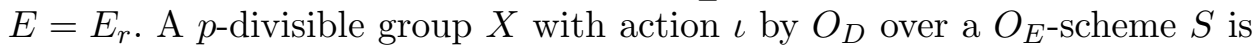
called an $r$-special $O_{D}$-module, if $X$ is of height $n^{2} d$ and $(X, \iota)$ satisfies the Kottwitz condition and the Eisenstein conditions relative to $r$.

Hence the previous proposition shows that a $r^{\circ}$-special formal $O_{D}$-module is just a special formal $O_{D}$-module in the sense of Drinfeld [5].

For the formulation of the moduli problem we will make use of the following lemma. The lemma follows from section 4, more precisely, Corollary 4.13. Alternatively, the lemma follows from the fact that $B(G,\{\mu\})$ has only one element, cf. [10], $\S 6$. Here $G=\operatorname{Res}_{F / \mathbb{Q}_{p}}\left(D^{\times}\right)$is the linear algebraic group over $\mathbb{Q}_{p}$ associated to $D^{\times}$, and $\{\mu\}$ is the conjugacy class of cocharacters with component $\left(1,0^{(n-1)}\right)$ for $\varphi_{0}$ and component $\left(1^{(n)}\right)$, resp. $\left(0^{(n)}\right)$ for $\varphi \neq \varphi_{0}$, depending on whether $r_{\varphi}=n$ or $r_{\varphi}=0$.

Lemma 2.5. Fix $r$. Let $\bar{k}$ be an algebraic closure of the residue field $\kappa_{E}$ of $O_{E}$. Any two $r$-special $p$-divisible groups over $\bar{k}$ are isogenous by a $O_{D}$-linear isogeny (which may be taken to be of height 0).

Now fix such a pair $\left(\mathbb{X}, \iota_{\mathbb{X}}\right)$ over $\bar{k}$. Denote by $O_{\breve{E}}$ the ring of integers in the completion of the maximal unramified extension of $E$. Then $\bar{k}$ is the residue field of $O_{\breve{E}}$. We consider the following set-valued functor $\mathcal{M}_{r}$ on Nilp $_{O_{\breve{E}}}$. It associates to $S \in$ Nilp $_{O_{\breve{E}}}$ the set of isomorphism classes of triples $(X, \iota, \varrho)$, where $(X, \iota)$ is an $r$-special $O_{D}$-module over $S$, and where

$$
\varrho: X \times_{S} \bar{S} \longrightarrow \mathbb{X} \times_{\operatorname{Spec} \bar{k}} \bar{S}
$$

is a $O_{D}$-linear quasi-isogeny of height zero. Here $\bar{S}=S \otimes_{O_{\breve{E}}} \bar{k}$. Our main conjecture can now be stated as follows.

Conjecture 2.6. ${ }^{2}$ The functor $\mathcal{M}_{r}$ is represented by $\hat{\Omega}_{F}^{n} \hat{\otimes}_{O_{F}} O_{\breve{E}}$.

Our main results towards this conjecture are the following.

\footnotetext{
${ }^{2}$ See the footnote 1.
} 
Theorem 2.7. The conjecture is true if $F / \mathbb{Q}_{p}$ is unramified.

If $F / \mathbb{Q}_{p}$ is ramified, we can still prove the following properties of $\mathcal{M}_{r}$ which are analogous to the properties of $\hat{\Omega}_{F}^{n} \hat{\otimes}_{O_{F}} O_{\breve{E}}$.

Theorem 2.8. The formal scheme $\mathcal{M}_{r}$ is flat over $\operatorname{Spf} O_{\breve{E}}$, and is $\pi$-adic. All its completed local rings are normal. Furthermore,

(i) there is an isomorphism between the special fibers

$$
\mathcal{M}_{r} \times_{\operatorname{Spf} O_{\breve{E}}} \operatorname{Spec} \bar{k} \simeq \hat{\Omega}_{F}^{n} \times_{\operatorname{Spf} O_{F}} \operatorname{Spec} \bar{k} .
$$

(ii) there is an isomorphism between the generic fibers

$$
\mathcal{M}_{r}^{\text {rig }} \simeq\left(\hat{\Omega}_{F}^{n} \times_{\operatorname{Spf} O_{F}} \operatorname{Spf} O_{\breve{E}}\right)^{\text {rig }} .
$$

Here the proof of point (ii) is due to P. Scholze.

We also prove the following variant of this theorem in the Lubin-Tate context. Let $F$ and $\varphi_{0}$ be as before, fix an integer $n \geq 2$ and let $r$ and $E=E_{r}$ have the same meaning as before. We now consider $p$-divisible formal groups $X$ of height $n d$ over $O_{E}$-schemes $S$ with an action $\iota: O_{F} \longrightarrow \operatorname{End}(X)$. We impose the following Kottwitz condition,

$$
\operatorname{char}(\iota(x) \mid \operatorname{Lie} X)=\prod_{\varphi}(T-\varphi(x))^{r_{\varphi}}, x \in O_{F} .
$$

In addition, we impose Eisenstein conditions (where the second condition in (2.9) is changed into

$$
\bigwedge^{2}\left(Q_{A_{\psi_{0}}}\left(\iota(\pi) \mid \operatorname{Lie}_{\psi_{0}} X\right)\right)=0
$$

see (8.2)).

We fix a pair $\left(\mathbb{X}, \iota_{\mathbb{X}}\right)$ over $\bar{k}$ as above. It is easy to see that $\mathbb{X}$ is unique up to $O_{F}$-linear isogeny. We may therefore define a functor $\mathcal{M}_{r}^{F}$ on $\operatorname{Nilp}_{O_{\breve{E}}}$ analogous to the functor $\mathcal{M}_{r}$ above. The formal scheme representing this functor will be denoted by the same symbol. If $r=r^{\circ}$ is the Drinfeld function, then $\mathcal{M}_{r^{\circ}}^{F}$ can be identified with the Lubin-Tate deformation space (this follows from Proposition 2.3). Our main result in this context is that this continues to hold for arbitrary $r$.

Theorem 2.9. The functor $\mathcal{M}_{r}^{F}$ is representable by $\operatorname{Spf} O_{\breve{E}}\left[\left[t_{1}, \cdots, t_{n-1}\right]\right]$. 


\section{The Kottwitz and Eisenstein conditions}

In this section, we analyze the conditions that can be put on a locally free module with $O_{D}$-action. We continue with the same notation as before. In addition, let $\tilde{E} \subset \overline{\mathbb{Q}}_{p}$ be a normal extension of $\mathbb{Q}_{p}$ which contains the images of all $\mathbb{Q}_{p}$-algebra homomorphisms $\tilde{F} \rightarrow \overline{\mathbb{Q}}_{p}$. We have $E \subset \tilde{E}$.

We denote by $\operatorname{Nrd}_{\varphi}: D \otimes_{F, \varphi} \tilde{E} \rightarrow \tilde{E}$ the reduced norm. Using it, we define the polynomial function

$$
\operatorname{Nrd}_{r}: D \otimes_{\mathbb{Q}_{p}} \tilde{E} \cong \prod_{\varphi} D \otimes_{F, \varphi} \tilde{E} \stackrel{\prod_{\varphi} \operatorname{Nrd}_{\varphi}^{r_{\varphi}}}{\longrightarrow} \tilde{E}
$$

If $\mathcal{M}$ is a quasicoherent sheaf on a scheme $S$ we denote by $\mathbb{V}_{S}(\mathcal{M})(T)=$ $\Gamma\left(T, \mathcal{M}_{T}\right)$ the corresponding flat sheaf on the category of $S$-schemes $T$. This sheaf is representable by a scheme over $S$ if $\mathcal{M}$ is a finite locally free $\mathcal{O}_{S^{-}}$ module.

We write simply $\mathbb{V}(D)$ for the affine space over $\mathbb{Q}_{p}$ associated to $D$. Then we may regard $\operatorname{Nrd}_{r}$ as a polynomial function (= morphism of schemes). It is defined over $E$,

$$
\operatorname{Nrd}_{r}: \mathbb{V}(D)_{E} \rightarrow \mathbb{A}_{E}^{1}
$$

Clearly this function is homogeneous of degree $\sum_{\varphi} n r_{\varphi}$.

Let $O_{D}$ be the ring of integers of $D$. Let $\tilde{F} \subset D$ be an unramified extension of $F$ of degree $n$. We denote by $\tau \in \operatorname{Gal}(\tilde{F} / F)$ the Frobenius automorphism. Then we may write

$$
O_{D}=O_{\tilde{F}}[\Pi], \quad \Pi^{n}=\pi, \quad \Pi a=\tau(a) \Pi, \text { for } a \in O_{\tilde{F}} .
$$

Here $\Pi$ is a prime element of $O_{D}$ and $\pi$ is a prime element of $O_{F}$.

We denote by $\mathbb{V}\left(O_{D}\right)$ the corresponding affine space over $\mathbb{Z}_{p}$. We will now define an integral version of (3.2).

We begin with a general remark. Let $S$ be an $O_{E}$-scheme and let $\mathcal{L}$ be a finite locally free $\mathcal{O}_{S}$-module with an action of $O_{D}$, i.e. a morphism of $\mathbb{Z}_{p}$-algebras

$$
\iota: O_{D} \rightarrow \operatorname{End}_{\mathcal{O}_{S}}(\mathcal{L})
$$

We will call $(\mathcal{L}, \iota)$ an $O_{D}$-module over $S$. 
If $T$ is an $S$-scheme and $\alpha \in \Gamma\left(T, O_{D} \otimes_{\mathbb{Z}_{p}} \mathcal{O}_{T}\right)$, we can take the determinant $\operatorname{det}\left(\alpha \mid \mathcal{L}_{T}\right)$. This defines a morphism

$$
\operatorname{det}_{\mathcal{L}}: \mathbb{V}\left(O_{D}\right)_{S} \rightarrow \mathbb{A}_{S}^{1}
$$

Let $\varphi \in \Phi$. We define an embedding

$$
O_{D} \otimes_{O_{F}, \varphi} O_{\tilde{E}} \rightarrow M\left(n \times n, O_{\tilde{E}}\right) .
$$

For this we choose an embedding $\tilde{\varphi}: \tilde{F} \rightarrow \overline{\mathbb{Q}}_{p}$ which extends $\varphi$ and define for $x \in \tilde{F}$,

$$
x \longmapsto\left(\begin{array}{cccc}
\tilde{\varphi}(x) & 0 & \ldots & 0 \\
0 & \tilde{\varphi}(\tau(x)) & \ldots & 0 \\
0 & 0 & \ldots & \tilde{\varphi}\left(\tau^{n-1}(x)\right)
\end{array}\right), \Pi \longmapsto\left(\begin{array}{ccccc}
0 & 1 & 0 & \ldots & 0 \\
0 & 0 & 1 & \ldots & 0 \\
& \ldots & & \\
\varphi(\pi) & 0 & 0 & \ldots & 0
\end{array}\right) .
$$

Consider the standard $M\left(n \times n, O_{\tilde{E}}\right)$-module $O_{\tilde{E}}^{n}$. Via restriction of scalars

$$
O_{D} \otimes_{\mathbb{Z}_{p}} O_{\tilde{E}} \rightarrow O_{D} \otimes_{O_{F}, \varphi} O_{\tilde{E}} \rightarrow M\left(n \times n, O_{\tilde{E}}\right),
$$

we obtain a $O_{D} \otimes_{\mathbb{Z}_{p}} O_{\tilde{E}}$-module $P_{\varphi}$. We define the $O_{D} \otimes_{\mathbb{Z}_{p}} O_{\tilde{E}}$-module

$$
P_{r}=\bigoplus P_{\varphi}^{r_{\varphi}}
$$

This module defines a polynomial function $\operatorname{Nrd}_{r}: \mathbb{V}\left(O_{D}\right)_{O_{\tilde{E}}} \rightarrow \mathbb{A}_{O_{\tilde{E}}}^{1}$,

$$
\operatorname{Nrd}_{r}(\xi)=\operatorname{det}\left(\xi \mid P_{r} \otimes_{O_{\tilde{E}}} R\right), \quad \xi \in O_{D} \otimes_{\mathbb{Z}_{p}} R
$$

where $R$ is an arbitrary $O_{\tilde{E}}$-algebra. Similarly, the module $P_{\varphi}$ defines a polynomial function $\operatorname{Nrd}_{\varphi}: \mathbb{V}\left(O_{D}\right)_{O_{\tilde{E}}} \rightarrow \mathbb{A}_{O_{\tilde{E}}}^{1}$. The polynomial function $\mathrm{Nrd}_{r}$ is invariant under $\operatorname{Gal}(\tilde{E} / E)$ and therefore defines a polynomial function

$$
\operatorname{Nrd}_{r}: \mathbb{V}\left(O_{D}\right)_{O_{E}} \rightarrow \mathbb{A}_{O_{E}}^{1}
$$

It follows from (3.5) that $\Pi$ acts on $P_{\varphi} \otimes_{O_{\tilde{E}}} \kappa_{\tilde{E}}$ as zero. Therefore the latter is a module over $\left(O_{D} / \Pi O_{D}\right) \otimes_{O_{\tilde{F}}} \kappa_{\tilde{E}}$. We see that the base change $\operatorname{Nrd}_{\varphi}$ factors through a polynomial function

$$
\left(\operatorname{Nrd}_{\varphi}\right)_{\kappa_{\tilde{E}}}: \mathbb{V}\left(O_{D} / \Pi O_{D}\right)_{\kappa_{\tilde{E}}} \rightarrow \mathbb{A}_{\kappa_{\tilde{E}}}^{1} .
$$

We obtain that $\left(\mathrm{Nrd}_{r}\right)_{\kappa_{\tilde{E}}}$ factors too,

$$
\left(\operatorname{Nrd}_{r}\right) \kappa_{\tilde{E}}: \mathbb{V}\left(O_{D} / \Pi O_{D}\right) \times_{\operatorname{Spec} \mathbb{F}_{p}} \operatorname{Spec} \kappa_{\tilde{E}} \rightarrow \mathbb{A}_{\kappa_{\tilde{E}}}^{1} .
$$


The affine algebra on the left hand side is an integral domain. Therefore $\left(\operatorname{Nrd}_{\varphi}\right)_{\kappa_{\tilde{E}}}$ is a non zero-divisor as an element of this algebra. This remains true after base change to any $\kappa_{\tilde{E}}$-algebra $R$.

Definition 3.1. Let $S$ be an $O_{E}$-scheme and let $(\mathcal{L}, \iota)$ be an $O_{D}$-module over $S$. We say that $(\mathcal{L}, \iota)$ satisfies the Kottwitz condition $\left(\mathbf{K}_{r}\right)$ with respect to $r$, if

$$
\operatorname{det}_{\mathcal{L}}=\left(\operatorname{Nrd}_{r}\right)_{S},
$$

where the right hand side is the base change with respect to $S \rightarrow \operatorname{Spec} O_{E}$.

Remark 3.2. By [9], Prop. 2.1.3, the condition is equivalent to the identity of polynomials in $\mathcal{O}_{S}[T]$ (comp. (2.2))

$$
\operatorname{char}(\iota(x) \mid \mathcal{L})=\prod_{\varphi} \varphi(\operatorname{chard}(x)(T))^{r_{\varphi}}, \quad \forall x \in O_{D} ;
$$

(this uses Amitsur's formula, comp. [4], Lemma 1.12).

It is clear that the Kottwitz condition is a closed condition. If the Kottwitz condition is fulfilled, we have

$$
\operatorname{rank}_{S} \mathcal{L}=n \sum_{\varphi} r_{\varphi}
$$

because $\operatorname{Nrd}_{r}$ is homogeneous of this degree.

Recall the maximal unramified subfield $F^{t} \subset F$ and its residue field $\kappa$. We denote by $\tilde{\kappa}$ the residue field of $\tilde{F}$. The maximal unramified subfield of $\tilde{F}$ is denoted by $\tilde{F}^{t}$. In addition to $\Psi=\operatorname{Hom}_{\mathbb{Q}_{p}}\left(F^{t}, \overline{\mathbb{Q}}_{p}\right)$, we introduce $\tilde{\Psi}=\operatorname{Hom}_{\mathbb{Q}_{p}}\left(\tilde{F}^{t}, \overline{\mathbb{Q}}_{p}\right)$.

We now introduce another condition which will turn out to be weaker than the Kottwitz condition. Let $R$ be an $O_{\tilde{E}^{-}}$-algebra. Let $(L, \iota)$ be an $O_{D^{-}}$ module over $R$. Then we have the decompositions

$$
L=\bigoplus_{\psi \in \Psi} L_{\psi}, \quad L=\bigoplus_{\tilde{\psi} \in \tilde{\Psi}} L_{\tilde{\psi}} .
$$

For example, the second of these decompositions is induced by

$$
O_{\tilde{F}^{t}} \otimes_{\mathbb{Z}_{p}} O_{\tilde{E}}=\prod_{\tilde{\psi} \in \tilde{\Psi}} O_{\tilde{E}} .
$$

For $\psi \in \Psi$ let $\Phi_{\psi}$ the set of all embeddings $\varphi: F \rightarrow \overline{\mathbb{Q}}_{p}$ whose restriction to $F^{t}$ is $\psi$. We define 


$$
r_{\psi}=\sum_{\varphi \in \Phi_{\psi}} r_{\varphi}
$$

Definition 3.3. Let $R$ be an $O_{\tilde{E}}$-algebra. We say that an $O_{D}$-module $(L, \iota)$ over $R$ satisfies the rank condition $\left(\mathbf{R}_{r}\right)$ with respect to $r$ if, for all $\tilde{\psi} \in \tilde{\Psi}$,

$$
\operatorname{rank}_{R} L_{\tilde{\psi}}=r_{\psi}
$$

where $\psi$ denotes the restriction of $\tilde{\psi}$ to $F^{t}$. We will write $r_{\tilde{\psi}}:=r_{\psi}$.

The rank condition is obviously an open condition (the rank goes up under specialization). It is also a closed condition since $\sum_{\tilde{\psi}} \operatorname{rank}_{R} L_{\tilde{\psi}}=$ rank $L$ is constant on the base. To check the rank condition it is enough to check it for the geometric points of $\operatorname{Spec} R$. If $R$ is an arbitrary $O_{E}$-algebra, we say that the rank condition is fulfilled, if it is fulfilled with respect to any base change $R \rightarrow \tilde{R}$ and an extension of the $O_{E}$-algebra structure to an $O_{\tilde{E}}$-algebra structure on $\tilde{R}$. The rank condition is then independent of the last choice.

Remark 3.4. The rank condition is independent of the choice of the chosen isomorphism (3.3). Indeed let $U \subset D$ be an unramified extension of degree $n$ of $F$. Then we could reformulate the rank condition using the action of $O_{U^{t}} \otimes_{\mathbb{Z}_{p}} O_{\tilde{E}}$. However, this yields the same condition. Indeed, since $U$ and $\tilde{F}$ are isomorphic field extensions of $F$, there is by Skolem-Noether an element $u \in D$, such that $u \tilde{F} u^{-1}=U$. Replacing $u$ by $u \Pi^{m}$ for a suitable integer $m$, we may assume that $u \in O_{D}^{\times}$. We denote by $L_{[u]}$ the $R$-module $L$ with the new $O_{D}$-action

$$
a \cdot{ }_{\text {new }} \ell=u a u^{-1} \ell .
$$

But the decomposition (3.9) for $L_{[u]}$,

$$
L_{[u]}=\bigoplus_{\tilde{\psi} \in \tilde{\Psi}}\left(L_{[u]}\right)_{\tilde{\psi}},
$$

is exactly the decomposition coming from the $O_{U^{t}}$-action on $L$. Since the multiplication by $u L \rightarrow L_{[u]}$ is an isomorphism of $O_{D}$-modules we obtain the independence.

We denote by $\kappa_{\tilde{E}}$ the residue class field of $O_{\tilde{E}}$. We consider a polynomial function over a reduced $\kappa_{\tilde{E}}$-algebra $R$,

$$
\chi: \mathbb{V}\left(O_{D}\right)_{R} \rightarrow \mathbb{A}_{R}^{1},
$$


which is multiplicative with respect to the ring structures of these schemes. We are given for each $R$-algebra $A$ a multiplicative map

$$
\chi_{A}: O_{D} / p O_{D} \otimes_{\mathbb{F}_{p}} A \rightarrow A .
$$

Let $a, b \in O_{D} / p O_{D} \otimes_{\mathbb{F}_{p}} A$. We claim that

$$
\chi((a+(\Pi \otimes 1) b))=\chi(a) .
$$

We regard this as an identity of polynomial functions on $\mathbb{V}\left(O_{D}\right)_{R} \times_{R} \mathbb{V}\left(O_{D}\right)_{R}$. We consider the units of $O_{D}$ as a subscheme $G \subset \mathbb{V}\left(O_{D}\right)_{R}$. This is dense in each fiber over $R$. Therefore it suffices to show (3.14) in the case where $a$ is a unit. By multiplicativity it suffices to show that

$$
\chi((1+(\Pi \otimes 1) b))=\chi(1) .
$$

We may restrict our attention to the universal case where $A=R\left[Y_{1}, \ldots, Y_{t}\right]$, which is also reduced.

We have

$$
(1+(\Pi \otimes 1) b)^{p^{s}}=1^{p^{s}}
$$

for some $p$-power $p^{s}$. Since $A$ is reduced and $\chi$ is multiplicative, we deduce (3.15).

Therefore (3.13) is equivalent to a functorial map

$$
O_{D} / \Pi O_{D} \otimes_{\mathbb{F}_{p}} A \rightarrow A
$$

We have $\tilde{\kappa}=O_{D} / \Pi O_{D}$. Therefore for each $\tilde{\psi} \in \tilde{\Psi}=\operatorname{Hom}_{\mathbb{F}_{p}}\left(\tilde{\kappa}, \kappa_{\tilde{E}}\right)=$ $\operatorname{Hom}_{\mathbb{Q}_{p}}\left(\tilde{F}^{t}, \overline{\mathbb{Q}}_{p}\right)$, we obtain a polynomial function

$$
\chi_{\tilde{\psi}}: O_{D} \rightarrow \tilde{\kappa} \stackrel{\tilde{\psi}}{\rightarrow} \kappa_{\tilde{E}} \rightarrow A .
$$

If Spec $R$ is connected and reduced, we deduce that the only multiplicative polynomial functions (3.12) have the form

$$
\prod_{\tilde{\psi}} \chi_{\tilde{\psi}}^{e_{\tilde{\psi}}}
$$

for suitable exponents $e_{\tilde{\psi}}$. These functions are also defined if $R$ is an arbitrary $\kappa_{\tilde{E}^{- \text {algebra. }}}$

Lemma 3.5. Let $R$ be a reduced $\kappa_{E}$-algebra. Let $(L, \iota)$ be an $O_{D}$-module over $R$. In particular $L$ is a finitely generated locally free $R$-module. 
Then the Kottwitz condition $\left(\mathbf{K}_{r}\right)$ for $(\mathcal{L}, \iota)$ is equivalent to the rank condition $\left(\mathbf{R}_{r}\right)$. For an arbitrary $O_{E}$-algebra $R$ the condition $\left(\mathbf{K}_{r}\right)$ implies the condition $\left(\mathbf{R}_{r}\right)$.

Proof. To prove this we make a base change $R \mapsto R \otimes_{O_{E}} O_{\tilde{E}}$ which is reduced if $R$ is a reduced $\kappa_{E}$-algebra. Therefore we may assume that $R$ is a $O_{\tilde{E}^{-}}$-algebra.

The first assertion follows because a polynomial function is uniquely determined by the numbers $e_{\tilde{\psi}}$ in (3.17).

The last assertion depends only on the geometric fibers. In this case we have either a $\kappa_{\tilde{E}^{-}}$-algebra or an $O_{E}$-algebra of characteristic 0 . We have already seen the first case. The characteristic 0 case is clear.

Proposition 3.6. Let us assume that $r=r^{\circ}$, i.e., $r_{\varphi}=0$ for $\varphi \neq \varphi_{0}$. Let $R$ be a $\kappa_{\tilde{E}}$-algebra. Let $(L, \iota)$ be a $O_{D}$-module over $R$. Then $(L, \iota)$ satisfies the condition $\left(\mathbf{K}_{r}\right)$ iff $\pi$ annihilates $L$ and the rank condition $\left(\mathbf{R}_{r}\right)$ is fulfilled.

The rank condition says in this case that for all $\tilde{\psi}: \tilde{\kappa} \rightarrow \kappa_{\tilde{E}}$

$$
\operatorname{rank} L_{\tilde{\psi}}=1, \text { if } \tilde{\psi}_{\mid F^{t}}=\psi_{0}=\varphi_{0 \mid F^{t}}, \quad \operatorname{rank} L_{\tilde{\psi}}=0 \text {, else. }
$$

Proof. By Lemma 3.5 we already know that the Kottwitz condition implies the rank conditions. If $a \in\left(O_{D} / p O_{D}\right) \otimes R$ we have $\operatorname{Nrd}_{r}(a \pi)=0$. Therefore the Kottwitz condition implies $\operatorname{det}\left(\pi \mid L_{\tilde{\psi}}\right)=0$. Since $L_{\tilde{\psi}}$ has rank 1 , this shows that $\pi$ annihilates $L$.

Assume conversely that the ranks are as indicated and that $\pi$ annihilates $L$. Then we deduce the result from the following Lemma.

Lemma 3.7. Let $R$ be a ring, such that $p R=0$. Let $n$ be a natural number. Let $L_{1}, \ldots, L_{n}$ be locally free $R$-modules of rank 1 . Assume we are given a chain of homomorphisms $\Pi_{i}: L_{i} \rightarrow L_{i+1}$ and $\Pi_{n}: L_{n} \rightarrow L_{1}$ such that

$$
\Pi_{n} \circ \Pi_{n-1} \circ \ldots \circ \Pi_{1}=0 .
$$

We set $L=L_{1} \bigoplus \ldots \oplus L_{n}$, and $\Pi=\Pi_{1} \bigoplus \ldots \oplus \Pi_{n}$. This is an endomorphism of $L$ such that $\Pi^{n}=0$.

Let $v \in M(n \times n, R)$ be a diagonal matrix. It induces an endomorphism $v: L \rightarrow L$. We consider an endomorphism of $L$ of the form

$$
v_{0}+v_{1} \Pi+v_{2} \Pi^{2}+\ldots+v_{n-1} \Pi^{n-1},
$$

where the $v_{i}$ are diagonal matrices.

Then

$$
\operatorname{det}_{F}\left(v_{0}+v_{1} \Pi+v_{2} \Pi^{2}+\ldots+v_{n-1} \Pi^{n-1}\right)=\operatorname{det}_{F} v_{0}
$$


Proof. We may assume $L_{i}=R$. Then $\Pi_{i}$ is the multiplication by some element $y_{i} \in R$. Our assumption says

$$
y_{n} y_{n-1} \cdot \ldots \cdot y_{1}=0 .
$$

One deduces that $\Pi^{n}=0$. We note that $\Pi v=v^{\prime} \Pi$, where $v^{\prime}$ is another diagonal matrix, which is obtained from $v$ by cyclically permuting the diagonal entries.

We may reduce to the universal case where $R$ is the quotient of a polynomial ring $R=\mathbb{F}_{p}\left[x_{i j}, y_{k}\right] / y_{1} \cdot \ldots \cdot y_{n}$, where $i \in[0, n-1], j \in[1, n], k \in[1, n]$. For fixed $i$, the $x_{i j}$ are the diagonal entries of $v_{i}$. In this case the ring $R$ is reduced.

Consider first the case where $v_{0}=E$ is the unit matrix. We take the universal case where the ring $R$ is reduced (as above but no indeterminates $x_{0 j}$ ). Then we have two commuting operators $\rho=v_{1} \Pi+v_{2} \Pi^{2}+\ldots+v_{n-1} \Pi^{n-1}$ and $v_{0}$. Therefore

$$
(E+\rho)^{p^{s}}=E^{p^{s}}+\rho^{p^{s}}
$$

But $\rho^{p^{s}}=0$ for $p^{s}>n$. This implies $\operatorname{det}(E+\rho)^{p^{s}}=1$. Because $R$ is reduced in the universal case, this implies (3.18) in the case where $v_{0}=E$. Clearly this shows also the case where $v_{0}$ is an invertible diagonal matrix.

In the general case we consider the universal $R$ as above and its localization,

$$
R \subset R\left[x_{01}^{-1}, x_{02}^{-1}, \ldots, x_{0 n}^{-1}\right] .
$$

Over the bigger ring $v_{0}$ becomes invertible and therefore the relation (3.18) holds. Hence it holds also over the subring $R$.

Let us recall the Eisenstein conditions. We recall the following notation: For an embedding $\psi: F^{t} \rightarrow \overline{\mathbb{Q}}_{p}$ we set

$$
\begin{aligned}
& A_{\psi}=\left\{\varphi: F \rightarrow \overline{\mathbb{Q}}_{p} \mid \varphi_{\mid F^{t}}=\psi, \text { and } r_{\varphi}=n\right\} \\
& B_{\psi}=\left\{\varphi: F \rightarrow \overline{\mathbb{Q}}_{p} \mid \varphi_{\mid F^{t}}=\psi, \text { and } r_{\varphi}=0\right\}
\end{aligned}
$$

We set $\psi_{0}=\varphi_{0 \mid F^{t}}$. This gives a partition of the set $\Phi_{\psi}$,

$$
\Phi_{\psi_{0}}=A_{\psi_{0}} \cup B_{\psi_{0}} \cup\left\{\varphi_{0}\right\}, \quad \Phi_{\psi}=A_{\psi} \cup B_{\psi} \text { for } \psi \neq \psi_{0} .
$$

Let $a_{\psi}=\left|A_{\psi}\right|, b_{\psi}=\left|B_{\psi}\right|$. Then we have

$$
a_{\psi}+b_{\psi}+\epsilon_{\psi}=e=\left[F: F^{t}\right],
$$

where $\epsilon_{\psi}=0$ if $\psi \neq \psi_{0}$, and $\epsilon_{\psi_{0}}=1$. 
We find (compare (3.10)) that

$$
r_{\psi}= \begin{cases}n a_{\psi}+1 & \text { if } \psi=\psi_{0} \\ n a_{\psi} & \text { if } \psi \neq \psi_{0}\end{cases}
$$

Let $Q(T) \in O_{F^{t}}[T]$ be the Eisenstein polynomial of a fixed prime element $\pi \in F$. We set $Q_{\psi}(T)=\psi(Q(T)) \in O_{E}[T]$. We set

$$
Q_{A_{\psi}}(T)=\prod_{\varphi \in A_{\psi}}(T-\varphi(\pi)), \quad Q_{B_{\psi}}(T)=\prod_{\varphi \in B_{\psi}}(T-\varphi(\pi)) .
$$

These are polynomials in $O_{E}[T]$. Moreover we set $Q_{0}(T)=T-\varphi_{0}(\pi)$. Then we have the decompositions

$$
\begin{aligned}
Q_{\psi}(T) & =Q_{A_{\psi}}(T) \cdot Q_{B_{\psi}}(T), \quad \text { for } \psi \neq \psi_{0} \\
Q_{\psi_{0}}(T) & =Q_{0}(T) \cdot Q_{A_{\psi_{0}}}(T) \cdot Q_{B_{\psi_{0}}}(T) .
\end{aligned}
$$

Let $R$ be an $O_{E}$-algebra. We will introduce the Eisenstein condition on a $O_{D}$-module $(L, \iota)$ over $R$. We have decompositions

$$
O_{F^{t}} \otimes_{\mathbb{Z}_{p}} O_{E} \cong \prod_{\psi \in \Psi} O_{E}, \quad O_{F^{t}} \otimes_{\mathbb{Z}_{p}} R \cong \prod_{\psi \in \Psi} R .
$$

This gives a decomposition

$$
L=\bigoplus_{\psi \in \Psi} L_{\psi}
$$

Definition 3.8. We say that $(L, \iota)$ satisfies the Eisenstein condition $\left(\mathbf{E}_{r}\right)$ with respect to $r$ if

$$
\begin{aligned}
\left(\left(Q_{0} \cdot Q_{A_{\psi_{0}}}\right)(\iota(\pi)) \mid L_{\psi_{0}}\right) & =0 \\
\bigwedge^{n+1}\left(Q_{A_{\psi_{0}}}\left(\iota(\pi) \mid L_{\psi_{0}}\right)\right) & =0, \\
\left(Q_{A_{\psi}}(\iota(\pi)) \mid L_{\psi}\right) & =0, \text { for } \psi \neq \psi_{0} .
\end{aligned}
$$

This definition applies to any $\mathcal{O}_{E}$-scheme $S$ and any $O_{D}$-module $(\mathcal{L}, \iota)$ over $S$.

Remarks 3.9. (i) The condition $\left(\mathbf{E}_{r}\right)$ only depends on the restriction to $O_{F}$ of the action $\iota$ of $O_{D}$ on $\mathcal{L}$. The condition depends on the choice of the uniformizer $\pi$. 
(ii) Let $r=r^{\circ}$, i.e., $r_{\varphi}=0$ for $\varphi \neq \varphi_{0}$. In this case $E=F$. We have two actions of $O_{F}$ on $\mathcal{L}$. The first is given by $O_{F} \stackrel{\varphi_{0}}{\longrightarrow} O_{E} \rightarrow \mathcal{O}_{S}$ and the second by $\iota$. By the Eisenstein condition these actions coincide. Indeed, $\mathcal{L}=\mathcal{L}_{\psi_{0}}$, and $a_{\psi_{0}}=0$ for all $\psi \in \Psi$. Therefore the Eisenstein condition (3.21) implies that $\iota(\pi)$ acts on $\mathcal{L}$ as $\varphi_{0}(\pi)$. The conditions (3.11) imply moreover that $\iota(a)$ for $a \in O_{F^{t}}$ acts via $\varphi_{0}(a)$ on $\mathcal{L}$.

(iii) Let $F / \mathbb{Q}_{p}$ be unramified, i.e., $F=F^{t}$. Then the first and the last Eisenstein conditions are empty. The second Eisenstein condition just says that $\operatorname{rank} \mathcal{L}_{\varphi_{0}} \leq n$.

(iv) Let us assume that $S$ is a $\kappa_{E}$-scheme. The images of the three polynomials $Q_{A_{\psi}}(T), Q_{B_{\psi}}(T)$ and $Q_{0}(T)$ in $\kappa_{E}[T]$ are respectively

$$
T^{a_{\psi}}, \quad T^{b_{\psi}}, \quad T .
$$

Therefore the Eisenstein conditions are in this case:

$$
\begin{aligned}
\iota(\pi)^{a_{\psi_{0}}+1} & =0 \text { on } \mathcal{L}_{\psi_{0}}, \\
\wedge^{n+1}\left(\iota(\pi)^{a_{\psi_{0}}}\right) & =0 \text { on } \wedge^{n+1} \mathcal{L}_{\psi_{0}}, \\
\iota(\pi)^{a_{\psi}} & =0 \text { on } \mathcal{L}_{\psi} \text { for } \psi \neq \psi_{0} .
\end{aligned}
$$

Definition 3.10. Let $(\mathcal{L}, \iota)$ be a $O_{D}$-module over an $O_{E}$-scheme $S$. We say that $(\mathcal{L}, \iota)$ satisfies the Drinfeld condition $\left(\mathbf{D}_{r}\right)$ with respect to $r$ if it satisfies the Eisenstein condition $\left(\mathbf{E}_{r}\right)$ and the rank condition $\left(\mathbf{R}_{r}\right)$.

The rank condition makes sense by the remark after Definition 3.3.

Lemma 3.11. Assume that $r=r^{\circ}$, i.e., $r_{\varphi}=0$ for $\varphi \neq \varphi_{0}$. Let $S$ be a $\kappa_{E}$-algebra.

Then the condition $\left(\mathbf{D}_{r}\right)$ implies $\left(\mathbf{K}_{r}\right)$.

This is a reformulation of Proposition 3.6.

\section{Formal $O_{D^{-} \text {-modules }}$}

Definition 4.1. Let $S$ be an $O_{E}$-scheme such that $p \mathcal{O}_{S}$ is a nilpotent ideal sheaf. A $p$-divisible $O_{D}$-module over $S$ is a $p$-divisible group $X$ of height $\left[D: \mathbb{Q}_{p}\right]=n^{2} d$ with an action

$$
\iota: O_{D} \rightarrow \text { End } X \text {. }
$$

In this case Lie $X$ is an $O_{D}$-module in the sense of (3.4). We say that $X$ satisfies $\left(\mathbf{K}_{r}\right)$ (3.7), resp. $\left(\mathbf{R}_{r}\right)$ (3.11), resp. $\left(\mathbf{E}_{r}\right)$ (3.21), resp. $\left(\mathbf{D}_{r}\right)$ (Definition $3.10)$ if the $\mathcal{O}_{S}$-module Lie $X$ with the $O_{D}$-action $d \iota$ does. 
A $p$-divisible $O_{D}$-module $X$ which satisfies $\left(\mathbf{D}_{r}\right)$ is called an $r$-special formal $O_{D}$-module. The name is justified because we will prove that $X$ has

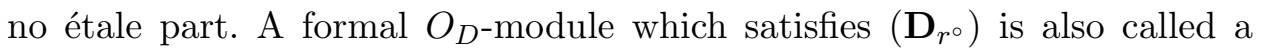
special formal $O_{D}$-module.

Remarks 4.2. (i) The definition of a special formal $O_{D}$-module above coincides with Drinfeld's definition in [5]. Indeed, in this case $r=r^{\circ}$, and it follows from Remarks 3.9, (ii) that $X$ is a strict formal $O_{F}$-module (the induced action of $O_{F}$ on $\operatorname{Lie} X$ is via the structure morphism $O_{F}=$ $\left.O_{E} \rightarrow \mathcal{O}_{S}\right)$.

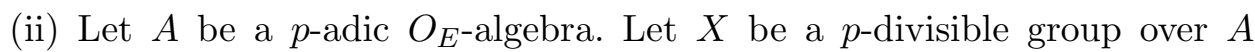
with an action (4.1). Then we define $\operatorname{Lie} X=\lim \operatorname{Lie} X \otimes A / p^{n}$. It is still a $O_{D}$-module and the definition above makes sense.

Assume that $p$ is locally nilpotent on $S$. We denote by $\mathbb{D}(X)$ the covariant crystal associated to $X$. The evaluation $\mathbb{D}(X)_{S}$ at $S$ coincides with the Lie algebra of the universal extension of $X$.

Let $a \in O_{D}$. We will often write simply $a$ when we mean the action $\iota(a)$ on $\mathbb{D}(X)_{S}$, or the derived action $d \iota(a)$ on $\operatorname{Lie} X$.

Proposition 4.3. Assume that $p \mathcal{O}_{S}$ is a locally nilpotent ideal sheaf. Let $X$ be a r-special formal $O_{D}$-module of height $n^{2} d$.

Then $\mathbb{D}(X)_{S}$ is locally on $S$ a free $O_{D} \otimes_{\mathbb{Z}_{p}} \mathcal{O}_{S}$-module of rank 1 . Moreover $X$ is a formal Lie group.

Proof. We begin with the case where $S=\operatorname{Spec} k$ where $k$ is a perfect field with a $\kappa_{\tilde{E}}$-structure. Let $W(k)$ be the ring of Witt vectors and $M=$ $\mathbb{D}(X)_{W(k)}$ be the covariant Dieudonné module.

We have a bijection of field embeddings

$$
\tilde{\Psi}=\operatorname{Hom}(\tilde{\kappa}, k)=\operatorname{Hom}\left(\tilde{F}^{t}, W(k) \otimes \mathbb{Q}\right) .
$$

We regard $\tilde{\psi} \in \tilde{\Psi}$ as a homomorphism $\tilde{\psi}: O_{\tilde{F}^{t}} \rightarrow W(k)$. We set

$$
M_{\tilde{\psi}}=\left\{m \in M \mid \iota(a) m=\tilde{\psi}(a) m, \text { for } a \in O_{\tilde{F}^{t}}\right\} .
$$

We have a direct decomposition

$$
M=\bigoplus M_{\tilde{\psi}}, \quad \tilde{\psi} \in \tilde{\Psi} .
$$

We denote by $\sigma$ the Frobenius acting on $W(k)$. The Verschiebung induces a map

$$
V: M_{\sigma \tilde{\psi}} \rightarrow M_{\tilde{\psi}}
$$


Therefore the rank of the $W(k)$-module $M_{\tilde{\psi}}$ is independent of $\tilde{\psi}$ and therefore equal to $n e$. For each $\tilde{\psi}: \tilde{F}^{t} \rightarrow W(k) \otimes \mathbb{Q}$ we have $\tilde{\psi} \tau=\sigma^{f} \tilde{\psi}$, where $\tau$ is from (3.3). Since $r_{\tilde{\psi}}$ depends only on the restriction of $\tilde{\psi}$ to $F^{t}$, we find $r_{\tilde{\psi}}=r_{\tilde{\psi} \tau}$. Obviously $\Pi$ induces a map

$$
\Pi: M_{\tilde{\psi} \tau} \rightarrow M_{\tilde{\psi}}
$$

We consider the commutative diagram

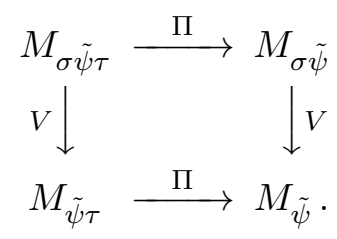

By the rank condition, the cokernels of the vertical maps are $k$-vector spaces of dimension $r_{\tilde{\psi} \tau}=r_{\tilde{\psi}}$. Therefore the lengths of the cokernels of both horizontal $\iota(\Pi)$ are also the same. On the other hand we have

$$
\sum_{\tilde{\psi}} \text { length } M_{\tilde{\psi}} / \Pi M_{\tilde{\psi} \tau}=\text { length } M / \Pi M=n f
$$

The last equality holds since by assumption length $M / p M=n^{2} d$. We conclude that

$$
\text { length } M_{\tilde{\psi}} / \Pi M_{\tilde{\psi} \tau}=1, \quad \text { for } \tilde{\psi} \in \tilde{\Psi}
$$

The last equation tells us that $M / \Pi M$ is a free $O_{D} / \Pi O_{D} \otimes_{\mathbb{F}_{p}} k$-module of rank 1. By Nakayama's lemma it follows that $M$ is a free $O_{D} \otimes_{\mathbb{Z}_{p}} W(k)$ module. This completes the proof of the first assertion in the case $S=\operatorname{Spec} k$.

To show that $X$ is a formal group we have to show that $V$ is nilpotent on $M / \iota(\Pi) M$. We consider the map induced by $V$ on the cokernels of the horizontal maps of the diagram (4.3). For each $\tilde{\psi}$ this map is either a bijection or it is zero. It suffices to see that this map is zero for some $\tilde{\psi}$. If not, we would have a bijection for each $\tilde{\psi}$. We denote the cokernels of the vertical maps by $\operatorname{Lie}_{\tilde{\psi}} X$. Then

$$
\operatorname{Lie} X=\bigoplus_{\tilde{\psi}} \operatorname{Lie}_{\tilde{\psi}} X
$$

We conclude that $\iota(\Pi): \operatorname{Lie}_{\tilde{\psi} \tau} X \rightarrow \operatorname{Lie}_{\tilde{\psi}} X$ is bijective for each $\tilde{\psi}$. This is a contradiction since $\iota(\Pi)$ is nilpotent on Lie $X$. Therefore $X$ is a formal group.

A base change argument shows that our result is true if $S$ is the spectrum of a field. In the general case we consider a point $s \in S$. We fix a basis $m$ of 
the $O_{D} \otimes \kappa(s)$-module $\mathbb{D}(X)_{S} \otimes \kappa(s)$. We may assume that $S=\operatorname{Spec} R$ and that $m$ is the image of an element $n \in \mathbb{D}(X)_{R}$. It follows from Nakayama's Lemma that the homomorphism induced by $n$

$$
O_{D} \otimes R \rightarrow \mathbb{D}(X)_{R}
$$

is an isomorphism in an open neighbourhood of $s$.

We state the following consequence separately:

Corollary 4.4. For each $\tilde{\psi} \in \tilde{\Psi}$ the cokernel of

$$
\Pi: \mathbb{D}(X)_{S, \tilde{\psi} \tau} \rightarrow \mathbb{D}(X)_{S, \tilde{\psi}}
$$

is a locally free $\mathcal{O}_{S}$-module of rank 1.

Proposition 4.5. Let $A$ be an $O_{E}$-algebra. Assume moreover that $A$ is a $p$-adic integral domain, with fraction field of characteristic 0 . Let $X$ be an $r$-special formal $O_{D}$-module over $A$.

Then Lie $X$ satisfies $\left(\mathbf{K}_{r}\right)$.

Proof. Let $K$ denote the fraction field of $A$. By Proposition $4.3, H=\mathbb{D}(X)_{K}$ is a free $O_{D} \otimes K$-module of rank 1 . We consider the decomposition

$$
H=\bigoplus_{\psi \in \Psi} H_{\psi} .
$$

We can assume that $A$ is a $O_{\tilde{E}}$-algebra. Let $\varphi \in \Phi$ be an extension of $\psi$. We write $\varphi \mid \psi$. Then $\pi$ acts semisimply on $H_{\psi}$ and has eigenvalues $\varphi(\pi)$ for $\varphi \mid \psi$, each with multiplicity $n^{2}$. Now $L=(\operatorname{Lie} X)_{K}$ is a quotient of $H$. It has the decomposition $L=\bigoplus L_{\psi}$. Assume first that $\psi \neq \psi_{0}$. By the Eisenstein condition, the eigenvalues of $\pi$ acting on $L_{\psi}$ are among $\varphi(\pi)$ with $\varphi \in A_{\psi}$. The multiplicity of the eigenvalues is at most $n^{2}$. But since by (3.11) $\operatorname{rank} L_{\psi}=n^{2} a_{\psi}$, each of these eigenvalues must have exactly multiplicity $n^{2}$. We assume now that $\psi=\psi_{0}$. Then again the eigenspaces of $\varphi(\pi)$ have dimension $\leq n^{2}$. But the second of the Eisenstein conditions says that for $\varphi=\varphi_{0}$ this multiplicity is $\leq n$. Since $\operatorname{rank} L_{\psi_{0}}=a_{\psi} n^{2}+n$, this implies that the multiplicity of $\varphi(\pi)$ is $n^{2}$ for $\varphi \in A_{\psi_{0}}$ and is $n$ for $\varphi=\varphi_{0}$. Altogether the multiplicity of the eigenvalue $\varphi(\pi)$ of $\pi$ acting on $L$ is $r_{\varphi} n$. This implies the Kottwitz condition on $L_{K}$. Since $\left(\mathbf{K}_{r}\right)$ is a closed condition, the assertion follows.

We will now assume that $S$ is a $\kappa_{\tilde{E}}$-scheme. The $O_{D}$-module $O_{D} \otimes \mathcal{O}_{S}$ defines a polynomial function 


$$
\rho: \mathbb{V}\left(O_{D}\right)_{S} \rightarrow \mathbb{A}_{S}^{1}
$$

The module $O_{D} \otimes \mathcal{O}_{S}$ has a composition series with factors

$$
O_{D} / \Pi O_{D} \otimes_{\tilde{\kappa}, \tilde{\psi}} \mathcal{O}_{S}
$$

where $\tilde{\psi} \in \tilde{\Psi}$. This last module defines the polynomial function $\chi_{\tilde{\psi}}$ from (3.16). One deduces easily that the determinant of the module $O_{D} \otimes \mathcal{O}_{S}$ is

$$
\rho=\prod_{\tilde{\psi} \in \tilde{\Psi}} \chi_{\tilde{\psi}}^{n e}=\prod_{\varphi \in \Phi} \operatorname{Nrd}_{\varphi}^{n} .
$$

Analogously to (4.2) we have a decomposition

$$
\mathbb{D}(X)_{S}=\bigoplus_{\tilde{\psi} \in \tilde{\Psi}} \mathbb{D}(X)_{S, \tilde{\psi}},
$$

where all summands are locally free $\mathcal{O}_{S}$-modules of rank $n e$. We set for $\psi \in \Psi$

$$
\mathbb{D}(X)_{S, \psi}=\bigoplus_{\tilde{\psi} \in \tilde{\Psi}} \mathbb{D}(X)_{S, \tilde{\psi}},
$$

where the sum runs over all $\tilde{\psi}$ such that $\tilde{\psi}_{\mid F^{t}}=\psi$. It follows from Proposition 4.3 that we have locally free $\mathcal{O}_{S}$-modules with ranks

$$
\operatorname{rank} \mathbb{D}(X)_{S, \tilde{\psi}} / \pi^{i} \mathbb{D}(X)_{S, \tilde{\psi}}=n i, \quad \text { for } 0 \leq i \leq e
$$

We note that the $\mathcal{O}_{S}$-modules $\mathbb{D}(X)_{S, \tilde{\psi}}$ are defined for each $O_{\tilde{E}}$-scheme with $p$ locally nilpotent.

Proposition 4.6. Let $(X, \iota)$ be a p-divisible $O_{D}$-module over a $\kappa_{E}$-scheme $S$. There are natural surjective maps

$$
\mathbb{D}(X)_{S, \psi} \rightarrow \operatorname{Lie}_{\psi} X, \quad \text { for } \psi \in \Psi
$$

(i) Assume that $(X, \iota)$ is r-special. Then the maps (4.4) induce isomorphisms

$$
\begin{aligned}
& \mathbb{D}(X)_{S, \psi} / \pi^{a_{\psi}} \mathbb{D}(X)_{S, \psi} \rightarrow \operatorname{Lie}_{\psi} X, \text { for } \psi \neq \psi_{0} \\
& \mathbb{D}(X)_{S, \psi_{0}} / \pi^{a_{\psi_{0}}} \mathbb{D}(X)_{S, \psi_{0}} \rightarrow \operatorname{Lie}_{\psi_{0}} X / \pi^{a_{\psi_{0}}} \operatorname{Lie}_{\psi_{0}} X
\end{aligned}
$$

In particular, the cokernel of any power of $\pi$ on Lie $X$ is a locally free $\mathcal{O}_{S}$-module. 
(ii) Conversely, assume that the following conditions on $(X, \iota)$ are satisfied.

1. Lie $X$ satisfies the rank condition $\left(\mathbf{R}_{r}\right)$.

2. The natural map $\mathbb{D}(X)_{S} \rightarrow \operatorname{Lie} X$ induces isomorphisms (4.5)

3. $\operatorname{Lie}_{\psi_{0}}$ is annihilated by $\pi^{a_{\psi_{0}}+1}$.

Then $X$ is r-special.

We will prove this together with the following Corollary:

Corollary 4.7. An $r$-special formal $O_{D}$-module $X$ over a $\kappa_{E}$-scheme $S$ satisfies the Kottwitz condition $\left(\mathbf{K}_{r}\right)$.

Remark 4.8. In the case where $r=r^{\circ}$, this corollary follows from Lemma 3.11.

Proof. Clearly we can restrict to the case where $S$ is a scheme over $\kappa_{\tilde{E}}$.

We first prove (i). The last condition of (3.22) says that for $\psi \neq \psi_{0}$ the first arrow of (4.5) exists. By (3.11) we have on both sides locally free $\mathcal{O}_{S}$-modules of the same rank. Therefore this arrow is an isomorphism.

For the second line in (4.5), we begin with the case where $S=$ Spec $k$. The second condition of (3.22) says that the rank of the following homomorphism of vector spaces

$$
\pi^{a_{\psi_{0}}}: \operatorname{Lie}_{\psi_{0}} X \rightarrow \operatorname{Lie}_{\psi_{0}} X
$$

is at most $n$. This shows

$$
\operatorname{dim}_{k}\left(\operatorname{Lie}_{\psi_{0}} X / \pi^{a_{\psi_{0}}} \operatorname{Lie}_{\psi_{0}} X\right) \geq \operatorname{dim}_{k} \operatorname{Lie}_{\psi_{0}} X-n=a_{\psi_{0}} n^{2} .
$$

Therefore the second arrow of (4.5) is an isomorphism because on the left hand side we have a vector space of dimension $n^{2} a_{\psi_{0}}$.

It follows that the $\mathcal{O}_{S}$-module $\operatorname{Lie}_{\psi_{0}} X / \pi^{a_{\psi_{0}}} \operatorname{Lie}_{\psi_{0}}$ has in each point of $S$ the same rank $n^{2} a_{\psi_{0}}$. This already proves assertion (i) in the case where $S$ is a reduced scheme.

The general case is a consequence of Lemma 4.9 below, applied to $L=$ $\operatorname{Lie}_{\psi_{0}} X, f=\pi^{a_{\psi_{0}}}, r=a_{\psi_{0}} n^{2}, m=a_{\psi_{0}} n^{2}+n$, and $s=n$.

Now we prove the corollary. We remark that the Kottwitz condition $\left(\mathbf{K}_{r}\right)$ is satisfied for Lie $X$. This is clear by the first isomorphism of (4.5) for the part $\operatorname{Lie}_{\psi} X$, for $\psi \neq \psi_{0}$. By the second isomorphism it suffices to show that the determinant morphism for the $O_{D}$-module $\pi^{a_{\psi_{0}}} \operatorname{Lie}_{\psi_{0}} X$ is $\operatorname{Nrd}_{\varphi_{0}}=\prod_{\tilde{\psi}} \chi_{\tilde{\psi}}$, where $\tilde{\psi}$ extends $\varphi_{0}$. But it follows from the isomorphism (4.5) and (3.11) that $\operatorname{rank} \pi^{a_{\psi_{0}}} \operatorname{Lie}_{\tilde{\psi}} X=1$ for each $\tilde{\psi} \in \tilde{\Psi}$ which extends $\varphi_{0}$. Therefore we conclude by Lemma 3.7. 
Now we prove (ii). We have to prove the Eisenstein conditions. For $\psi \neq \psi_{0}$ they are clear. We denote by $K_{\psi_{0}}$ the kernel of the natural map $\mathbb{D}(X)_{S, \psi_{0}} \rightarrow \operatorname{Lie}_{\psi_{0}} X$. We obtain $\pi^{a_{\psi_{0}}} \mathbb{D}(X)_{S, \psi_{0}} \supset K_{\psi_{0}} \supset \pi^{a_{\psi_{0}}+1} \mathbb{D}(X)_{S, \psi_{0}}$. Then the rank condition shows that

$$
\pi^{a_{\psi_{0}}} \mathbb{D}(X)_{S, \psi_{0}} / K_{\psi_{0}}=\pi^{a_{\psi_{0}}} \operatorname{Lie}_{\psi_{0}} X
$$

has rank $n$. This proves the second condition of (3.22). The other Eisenstein conditions are trivially satisfied.

In the preceding proof, we used the following lemma.

Lemma 4.9. Let $R$ be a local ring with residue field $k$. Let $L$ be a finitely generated free $R$-module of rank $m$. Let $f: L \rightarrow L$ be an endomorphism.

Let $r=\operatorname{dim}_{k}(L / f(L)) \otimes k$ and let $s=m-r$. We assume that

$$
\bigwedge^{s+1} f=0 .
$$

Then $L / f(L)$ is a free $R$-module of rank $r$.

Proof. The exact sequence

$$
L \otimes k \rightarrow L \otimes k \rightarrow(L / f(L)) \otimes k \rightarrow 0
$$

shows that there is a basis of $L \otimes k$ of the form

$$
f\left(\bar{y}_{1}\right), \ldots, f\left(\bar{y}_{s}\right), \bar{e}_{1}, \ldots, \bar{e}_{r}
$$

where $\bar{y}_{1}, \ldots, \bar{y}_{s}, \bar{e}_{1}, \ldots, \bar{e}_{r} \in L \otimes k$, and where the images of $\bar{e}_{1}, \ldots, \bar{e}_{r}$ in $(L / f(L)) \otimes k$ form a basis.

Lifting the elements $\bar{y}_{1}, \ldots, \bar{y}_{s}, \bar{e}_{1}, \ldots, \bar{e}_{r}$ to $L$ we obtain a basis of this $R$-module,

$$
f\left(y_{1}\right), \ldots, f\left(y_{s}\right), e_{1}, \ldots e_{r} .
$$

The elements $\bar{y}_{1}, \ldots, \bar{y}_{s} \in L \otimes k$ are linearly independent. Therefore we find a second basis of $L$,

$$
y_{1}, \ldots, y_{s}, x_{1}, \ldots, x_{r}
$$

We write the matrix of $f$ with respect to the basis (4.6) and (4.7) as a $(s+r) \times(s+r)$ block matrix:

$$
\left(\begin{array}{cc}
E_{s} & A \\
\mathbf{0} & B
\end{array}\right)
$$


The assumption $\bigwedge^{s+1} f=0$ implies that the matrix $B$ is zero. We find

$$
f\left(x_{i}\right)=\sum_{j} a_{j i} f\left(y_{j}\right), \quad a_{j i} \in R .
$$

We set $z_{i}=x_{i}-\sum_{j} a_{j i} y_{j} \in \operatorname{Ker} f$. Clearly

$$
y_{1}, \ldots, y_{s}, z_{1}, \ldots, z_{r}
$$

is also a basis of $L$, i.e., we may assume WLOG that $x_{i}=z_{i}$. But then the matrix of $f$ becomes

$$
\left(\begin{array}{cc}
E_{s} & \mathbf{0} \\
\mathbf{0} & \mathbf{0}
\end{array}\right)
$$

From this our assertion is obvious.

Before continuing, we add a lemma needed later which is proved in the same manner.

Lemma 4.10. Let $n, m$, and $r$ be natural numbers. Let $R$ be a commutative ring. Let $W$ be a locally free $R$-module of rank $n$. Let $f: W \rightarrow W$ be an endomorphism such that Coker $f$ is a locally free $R$-module of rank $r$.

Let $V \subset W$ be a direct summand of rank $m$. We assume that $s=m-r \geq 0$ and that

$$
\bigwedge^{s+1}\left(f_{\mid V}\right)=0
$$

Then $\operatorname{Ker} f \subset V$.

Proof. We note that the assumptions of the lemma are compatible with base change $R \rightarrow S$. The situation of the lemma is always defined over a noetherian subring. Therefore we may assume that $R$ is noetherian. The desired inclusion may be checked over the localizations of $R$. Therefore we may assume that $R$ is a local noetherian ring with maximal ideal $\mathfrak{m}$. Finally the matrix of Ker $f \rightarrow W / V$ is zero if it is zero modulo $\mathfrak{m}^{t}$ for all $t \in \mathbb{N}$. Therefore we may assume that $R$ is an artinian local ring. We also note that under the assumptions $\operatorname{Ker} f$ is a direct summand of $W$ of rank $r$.

If $R$ is a field, the assumption of the lemma implies rank $f_{\mid V} \leq s$. Hence $\operatorname{dim} \operatorname{Ker} f_{\mid V} \geq r=\operatorname{dim} \operatorname{Ker} f$. This implies Ker $f_{\mid V}=\operatorname{Ker} f$ and the lemma.

Now let $(R, \mathfrak{m})$ be any artinian local ring. Let $e_{1}, \ldots, e_{r}$ be a basis of Ker $f$. It follows from the case of a field that $V$ has a basis of the form

$$
v_{1}, \ldots, v_{s}, e_{1}+\rho_{1}, \ldots, e_{r}+\rho_{r},
$$


where $\rho_{i} \in \mathfrak{m} W$. If the lemma is false we can choose $t \in \mathbb{N}$ maximal such that there is a basis of the form (4.8) with $\rho_{i} \in \mathfrak{m}^{t} W$.

By assumption $f\left(v_{1}\right), \ldots, f\left(v_{s}\right)$ are linearly independent modulo $\mathfrak{m}$. Therefore we find a basis of $V$ of the form

$$
f\left(v_{1}\right), \ldots, f\left(v_{s}\right), u_{1}, \ldots, u_{r} .
$$

We write the matrix of $f_{\mid V}$ with respect to the matrices (4.8) and (4.9) as a block matrix,

$$
\left(\begin{array}{cc}
E_{s} & * \\
\mathbf{0} & X
\end{array}\right)
$$

By the assumption $\wedge^{s+1}\left(f_{\mid V}\right)=0$ all determinants of $(s+1) \times(s+1)$ minors of this matrix are zero. Therefore the matrix $X$ is zero. We obtain equations

$$
f\left(\rho_{i}\right)=f\left(e_{i}+\rho_{i}\right)=\sum_{j} a_{j i} f\left(v_{j}\right) .
$$

Since the left hand side is in $\mathfrak{m}^{t} W$ we conclude that $a_{j i} \in \mathfrak{m}^{t}$. We find $\rho_{i}-\sum_{j} a_{j i} v_{j} \in \operatorname{Ker} f$. We may write

$$
\rho_{i}-\sum_{j} a_{j i} v_{j}=\sum_{k} c_{k i} e_{k}
$$

where necessarily $c_{k i} \in \mathfrak{m}^{t}$. The last equation gives:

$$
\left(e_{i}+\rho_{i}\right)-\sum_{j} a_{j i} v_{j}-\sum_{k} c_{k i}\left(e_{k}+\rho_{k}\right)=e_{i}-\sum_{k} c_{k i} \rho_{k} .
$$

The RHS is an element of $V$. But this shows that we may replace in (4.8) the element $e_{i}+\rho_{i}$ by $e_{i}+\rho_{i}^{\prime}$ with $\rho_{i}^{\prime}=\sum_{k} c_{k i} \rho_{k} \in \mathfrak{m}^{2 t} W$. This is a contradiction.

Remark 4.11. If $R$ is a local ring such that each element of the maximal ideal $\mathfrak{m}$ is nilpotent, we can replace the condition that "Coker $f$ is a free $R$-module of rank $r$ " by the weaker assumption that "Ker $f \subset W$ is a direct summand of rank $r$ ". Indeed, over $R$ any free submodule of a free module is a direct summand. This shows that Coker $f$ is free. The weaker assumption also suffices if $R$ is a ring such that $R \rightarrow \prod R_{\mathfrak{p}}$ is injective, where $\mathfrak{p}$ runs over all minimal prime ideals of $R$, since then we may reduce to $R=R_{\mathfrak{p}}$.

We will now study the display of a formal $O_{D}$-module over a $\kappa_{E}$-scheme $S$ which satisfies $\left(\mathbf{D}_{r}\right)$. To ease the notation we will assume that $S=\operatorname{Spec} R$. 
We denote by $\mathcal{P}=(P, Q, F, \dot{F})$ the display of $X$. We use the notation $I:=$ $I_{R}:=\operatorname{ker}(W(R) \rightarrow R)$. Recall that $\mathbb{D}(X)_{R}=P / I P$.

Again write $\Psi=\operatorname{Hom}\left(\kappa, \kappa_{E}\right)$ for the set of field embeddings. We obtain the decompositions

$$
P=\bigoplus_{\psi \in \Psi} P_{\psi}, \quad Q=\bigoplus_{\psi \in \Psi} Q_{\psi}
$$

By Proposition 4.6 we obtain:

$$
\begin{aligned}
\pi^{a_{\psi_{0}}+1} P_{\psi_{0}}+I P_{\psi_{0}} \subset Q_{\psi_{0}} & \subset \pi^{a_{\psi_{0}}} P_{\psi_{0}}+I P_{\psi_{0}}, \\
Q_{\psi} & =\pi^{a_{\psi}} P_{\psi}+I P_{\psi}, \quad \text { for } \psi \neq \psi_{0} .
\end{aligned}
$$

The maps $F$ and $\dot{F}$ induce maps

$$
F_{\psi}: P_{\psi} \rightarrow P_{\psi \sigma}, \quad \dot{F}_{\psi}: P_{\psi} \rightarrow P_{\psi \sigma}
$$

Here $\sigma$ denotes the Frobenius automorphism of $F^{t}$ over $\mathbb{Q}_{p}$. We set $Q_{\psi}^{\prime}=P_{\psi}$ for $\psi \neq \psi_{0}$ and we define $Q_{\psi_{0}}^{\prime}$ to be the unique submodule of $P_{\psi_{0}}$ such that $P_{\psi_{0}} \supset Q_{\psi_{0}}^{\prime} \supset Q_{\psi_{0}}$ and such that $Q_{\psi_{0}}^{\prime} / Q_{\psi_{0}}$ is the kernel of the homomorphism

$$
\pi^{a_{\psi_{0}}}: P_{\psi_{0}} / Q_{\psi_{0}} \rightarrow P_{\psi_{0}} / Q_{\psi_{0}}
$$

By Lemma 4.9 we know that this kernel is a direct summand of $P_{\psi_{0}} / Q_{\psi_{0}}$.

We set

$$
Q^{\prime}=\bigoplus_{\psi} Q_{\psi}^{\prime}, \quad F_{\psi}^{\prime}=F_{\psi} \pi^{a_{\psi}}, \quad \dot{F}_{\psi}^{\prime}=\dot{F}_{\psi} \pi^{a_{\psi}} .
$$

We obtain Frobenius-linear homomorphisms

$$
F^{\prime}=\bigoplus_{\psi} F_{\psi} \pi^{a_{\psi}}: P \rightarrow P, \quad \dot{F}=\bigoplus_{\psi} \dot{F}_{\psi} \pi^{a_{\psi}}: Q^{\prime} \rightarrow P .
$$

We claim that the quadruple $\mathcal{P}^{\prime}=\left(P, Q^{\prime}, F^{\prime}, \dot{F}^{\prime}\right)$ is the display of a special formal $O_{D}$-module.

Theorem 4.12. Let $R$ be a $\kappa_{E}$-algebra. We assume that the nilradical of $R$ is a nilpotent ideal. Let $\mathcal{C}_{r, R}$ be the category of $r$-special formal $O_{D}$-modules, and let $\mathcal{C}_{\mathbf{0}, R}$ the category of special formal $O_{D}$-modules (Definition 4.1).

The construction $\mathcal{P} \mapsto \mathcal{P}^{\prime}$ is an equivalence of categories

$$
\mathcal{C}_{r, R} \rightarrow \mathcal{C}_{\mathbf{0}, R}
$$


Proof. We begin with the case $S=\operatorname{Spec} k$, where $k$ is a perfect field. Let the covariant Dieudonné $M_{X}$ be identified with $P$. In this case (4.10) is equivalent with

$$
\begin{aligned}
& \pi^{a_{\psi_{0}}+1} M_{\psi_{0}, X} \subset V M_{\psi_{0} \sigma, X} \subset \pi^{a_{\psi_{0}}} M_{\psi_{0}}, \\
& V M_{\psi \sigma, X}=\pi^{a_{\psi}} M_{\psi, X} \quad \text { for } \psi \neq \psi_{0} .
\end{aligned}
$$

We define

$$
\begin{gathered}
V^{\prime}=\pi^{-a_{\psi}} V: M_{\psi \sigma, X} \rightarrow M_{\psi, X} \\
F^{\prime}=\pi^{a_{\psi}} F: M_{\psi, X} \rightarrow M_{\psi \sigma, X} .
\end{gathered}
$$

Then the Dieudonné module $\left(M_{X}, F^{\prime}, V^{\prime}\right)$ corresponds to the display above. From this we see that $\mathcal{P}^{\prime}$ is the Dieudonné module of a special formal $O_{D}$-module. Indeed, by the remark after (3.22) we need only to verify $\left(\mathbf{R}_{r}\right)$ (3.11) for $\mathcal{P}^{\prime}$. But this follows easily from (3.19) and (4.13).

If conversely $\left(M, F^{\prime}, V^{\prime}\right)$ is the Dieudonné module of a special formal $O_{D}$-module, then we find

$$
F^{\prime} M_{\psi_{0}, X} \subset \pi^{e-1} M_{\psi_{0} \sigma, X}, \quad F^{\prime} M_{\psi, X} \subset \pi^{e} M_{\psi \sigma, X} \text {, for } \psi \neq \psi_{0} .
$$

This follows because $V^{\prime} M_{\psi \sigma}=M_{\psi}$ for $\psi \neq \psi_{0}$ and $M_{\psi_{0}} / V^{\prime} M_{\psi_{0} \sigma}$ is annihilated by $\pi$. Therefore the formulas $V=\pi^{a_{\psi}} V^{\prime}$ and $F=\pi^{-a_{\psi}} F^{\prime}$ define a Dieudonné module structure on $M$ such that (4.13) is satisfied. This shows that $(M, F, V)$ is the Dieudonné module of an $r$-special formal $O_{D}$-module. This proves the theorem in the case of a perfect field.

In the general case we need first to verify that $\mathcal{P}^{\prime}$ is a display. The only non-trivial property is that $\dot{F}^{\prime}$ is a Frobenius-linear epimorphism. To show this, we take locally on Spec $R$ a normal decomposition of $\mathcal{P}^{\prime}$ and consider the matrix of $F^{\prime} \oplus \dot{F}^{\prime}$. We have to show that the image of the determinant of this matrix in $R$ is a unit. But this property follows since we know it for a perfect field. The same argument shows that $\mathcal{P}^{\prime}$ is nilpotent. Therefore we have defined a functor (4.12).

We construct first a quasi-inverse functor in the case that the ring $R$ is reduced. Let $\mathcal{P}^{\prime}$ be the display of a special formal $O_{D}$-module. We note that $P^{\prime} / I P^{\prime}$ is a locally free $O_{D} \otimes R$-module of rank 1 . In particular, it has a filtration by direct summands as $R$-modules,

$$
0=\pi^{e}\left(P_{\psi}^{\prime} / I P_{\psi}^{\prime}\right) \subset \pi^{e-1}\left(P_{\psi}^{\prime} / I P_{\psi}^{\prime}\right) \subset \ldots \subset \pi\left(P_{\psi}^{\prime} / I P_{\psi}^{\prime}\right) \subset P_{\psi}^{\prime} / I P_{\psi}^{\prime}
$$

The multiplication by $\pi$ gives an isomorphism between the subquotients of this filtration. 
If we have a direct $R$-module summand $L \subset P_{\psi}^{\prime} / I P_{\psi}^{\prime}$ with the property that $\pi\left(P_{\psi}^{\prime} / I P_{\psi}^{\prime}\right) \subset L \subset P_{\psi}^{\prime} / I P_{\psi}^{\prime}$, we obtain therefore a direct $R$-module summand $\pi^{a_{\psi}+1}\left(P_{\psi}^{\prime} / I P_{\psi}^{\prime}\right) \subset \pi^{a_{\psi}} L \subset \pi^{a_{\psi}} P_{\psi}^{\prime} / I P_{\psi}^{\prime}$.

This gives the possibility to invert our construction $\mathcal{P} \rightarrow \mathcal{P}^{\prime}$. We set $P=P^{\prime}$. We note that $Q_{\psi}^{\prime}=P_{\psi}^{\prime}$ if $\psi \neq \psi_{0}$. We set in general

$$
Q_{\psi}=\pi^{a_{\psi}} Q_{\psi}^{\prime}+I P_{\psi}
$$

We want to define $F$ and $\dot{F}$ by the formulas

$$
F_{\psi}=\pi^{-a_{\psi}} F_{\psi}^{\prime}, \quad \dot{F}_{\psi}=\pi^{-a_{\psi}} \dot{F}_{\psi}^{\prime} .
$$

We note that $F_{\psi}^{\prime} Q_{\psi}^{\prime}=p \dot{F}_{\psi}^{\prime} Q_{\psi}^{\prime}$. This implies for $\psi \neq \psi_{0}$ that $F_{\psi}^{\prime} P_{\psi} \subset \pi^{e} P_{\psi \sigma}$. From $\pi P_{\psi_{0}}^{\prime} \subset Q_{\psi_{0}}^{\prime}$ we conclude that $F_{\psi_{0}}^{\prime} P_{\psi_{0}} \subset \pi^{e-1} P_{\psi_{0} \sigma}$. Since $R$ is reduced, $\pi$ operates injectively on $W(R)$ and therefore the definition of $F_{\psi}$ makes sense. From (4.15) we see that also the definition of $\dot{F}$ makes sense. We have to show that $\mathcal{P}=(P, Q, F, \dot{F})$ is indeed a display. But this follows from the case of a perfect field treated above.

Now we treat the case of a nonreduced ring $R$. We assume that we have a divided power thickening $R \rightarrow S$, and that the theorem is already known for $S$. We denote by $X$ an $r$-special formal $O_{D}$-module over $S$, and by $X^{\prime}$ the corresponding special formal $O_{D}$-module over $S$. We show that our functor gives a bijection between the liftings of $X$ to an $r$-special formal $O_{D}$-module over $R$ and the liftings of $X^{\prime}$ to a special formal $O_{D}$-module over $R$. This will prove the theorem by induction. By Grothendieck-Messing, the liftings of $X$ correspond to liftings of the Hodge-filtration,

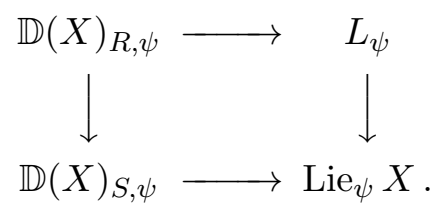

If $\psi \neq \psi_{0}$ we have no choice for $L_{\psi}$ because, by Proposition 4.6, $L_{\psi}=$ $\mathbb{D}(X)_{R, \psi} / \pi^{a_{\psi}} \mathbb{D}(X)_{R, \psi}$. As a special case this holds also for $X^{\prime}$. Now let $\psi=\psi_{0}$. Let $\bar{Q}_{R}$ and $\bar{Q}_{S}$ the kernels of the two horizontal maps in (4.16). Then we have $\pi^{a_{\psi}} \mathbb{D}(X)_{R, \psi} \supset \bar{Q}_{R} \supset \pi^{a_{\psi}+1} \mathbb{D}(X)_{R, \psi}$. We replace $\bar{Q}_{R}$ by $\bar{Q}_{R}^{\prime}:=\pi^{-a_{\psi}} \bar{Q}_{R}$. This makes sense because we have a bijection

$$
\mathbb{D}(X)_{R, \psi} / \pi \mathbb{D}(X)_{R, \psi} \stackrel{\pi^{a_{\psi}}}{\longrightarrow} \pi^{a_{\psi}} \mathbb{D}(X)_{R, \psi} / \pi^{a_{\psi}+1} \mathbb{D}(X)_{R, \psi} .
$$


Then $L^{\prime}=\mathbb{D}(X)_{R, \psi} / \bar{Q}_{R}^{\prime}$ is a lifting of $\operatorname{Lie}_{\psi} X^{\prime}$ which defines a lifting of the special formal $O_{D}$-module $X^{\prime}$. This sets up the desired bijection of liftings.

Corollary 4.13. Let $k$ be an algebraically closed field which is at the same time a $\kappa_{E}$-algebra. Any two $r$-special formal $O_{D}$-modules over $k$ are isogenous by a $O_{D}$-linear isogeny.

Proof. By Theorem 4.12, we are reduced to the case of special formal $O_{D^{-}}$ modules, i.e., the case $r=r^{\circ}$. In this case, the assertion follows from [5], 2 , comp. [3], Prop. 5.2.

Let $\breve{E}$ the completion of the maximal unramified extension of $E$. Its residue class field $\bar{k}$ is an algebraic closure of $\kappa_{E}$. We fix an $r$-special formal $O_{D}$-module $\left(\mathbb{X}, \iota_{\mathbb{X}}\right)$ over $\bar{k}$ (a framing object).

Definition 4.14. We define the set-valued functor $\mathcal{M}_{r}$ on the category of $O_{\breve{E}}$-schemes as follows ${ }^{3}$. Then $\mathcal{M}_{r}$ associates to scheme $S \in\left(\operatorname{Sch} / O_{\breve{E}}\right)$ the set of isomorphism classes of triples $(X, \iota, \rho)$. Here $(X, \iota)$ is an $r$-special formal $O_{D}$-module over $S$, and $\rho$ denotes a $O_{D}$-linear isogeny $X \times_{\operatorname{Spec}} O_{\breve{E}}$ $\operatorname{Spec} \bar{k} \rightarrow \mathbb{X} \times_{\text {Spec } \bar{k}} S$ of height zero.

We write $\overline{\mathcal{M}}_{r}$ for the restriction of this functor to $\bar{k}$-schemes $S$. Theorem 4.12 now implies the following corollary.

Corollary 4.15. The functor $\overline{\mathcal{M}}_{r}$ is representable by a scheme over $\bar{k}$ which is isomorphic to $\overline{\mathcal{M}}_{r}$. Hence there is an isomorphism $\overline{\mathcal{M}}_{r} \simeq \hat{\Omega}^{n} \otimes_{O_{\breve{E}}} \bar{k}$.

Proof. The isomorphism $\overline{\mathcal{M}}_{r} \simeq \overline{\mathcal{M}}_{r}$ 。 follows from Theorem 4.12. The last assertion follows from [5], which also implies that $\overline{\mathcal{M}}_{r}$ is a scheme.

\section{The local model}

In this section we consider the local structure of the formal scheme $\mathcal{M}_{r}$ (Definition 4.14). By the general theory [18], this comes down to considering the local model of $\mathcal{M}_{r}$. Let us define it.

Recall that $D$ is the central division algebra with invariant $1 / n$ over $F$. Let $V$ be a $D$-vector space of dimension 1 and let $\Lambda$ be an $O_{D}$-lattice in $V$.

The local model in question represents the following functor on $\left(\mathrm{Sch} / O_{E}\right)$ :

\footnotetext{
${ }^{3}$ We will prove in Proposition 5.8 that this definition coincides with the one in section 2 .
} 


$$
\begin{aligned}
\mathbb{M}_{r}(S)=\left\{\mathcal{F} \subset \Lambda \otimes_{\mathbb{Z}_{p}} \mathcal{O}_{S} \mid\right. & O_{D} \text {-stable } \mathcal{O}_{S} \text {-submodule, locally on } S \text { a } \\
& \text { direct summand, }\left(\Lambda \otimes_{\mathbb{Z}_{p}} \mathcal{O}_{S}\right) / \mathcal{F} \text { satisfies } \\
& \text { conditions } \left.\left(\mathbf{R}_{r}\right) \text { and }\left(\mathbf{E}_{r}\right)\right\} .
\end{aligned}
$$

Lemma 5.1. The functor $\mathbb{M}_{r}$ is representable by a projective scheme over Spec $O_{E}$. The geometric generic fiber is isomorphic to $\mathbb{P}^{n-1}$.

Let $S$ be an E-scheme. Consider a $\mathcal{O}_{S}$-submodule $\mathcal{F} \subset \Lambda \otimes_{\mathbb{Z}_{p}} \mathcal{O}_{S}$ which is locally a direct summand and which is $O_{D}$-stable. Then $\left(\Lambda \otimes_{\mathbb{Z}_{p}} \mathcal{O}_{S}\right) / \mathcal{F}$ satisfies conditions $\left(\mathbf{R}_{r}\right)$ and $\left(\mathbf{E}_{r}\right)$ if and only if it satisfies the condition $\left(\mathbf{K}_{r}\right)$.

Proof. The first assertion is obvious since the rank condition is a closed condition, cf. the remark after Definition 3.3.

The implication " $\Rightarrow$ " in the last assertion follows as in Proposition 4.5. To show the converse, let $R$ be a $\overline{\mathbb{Q}}_{p}$-algebra and $S=\operatorname{Spec} R$. Let $\mathcal{F}$ be a direct summand of $\Lambda \otimes_{\mathbb{Z}_{p}} R$ that is $O_{D^{-}}$-stable and such that $\left(\Lambda \otimes_{\mathbb{Z}_{p}} \mathcal{O}_{S}\right) / \mathcal{F}$ satisfies $\left(\mathbf{K}_{r}\right)$. There are decompositions

$$
\Lambda \otimes_{\mathbb{Z}_{p}} R=\bigoplus_{\varphi} \Lambda_{\varphi}, \quad \mathcal{F}=\bigoplus_{\varphi} \mathcal{F}_{\varphi},
$$

where $\varphi$ runs through the embeddings of $F$ into $\overline{\mathbb{Q}}_{p}$. Here $O_{F}$ acts on the summand corresponding to $\varphi$ via $\varphi: F \rightarrow \overline{\mathbb{Q}}_{p} \rightarrow R$. Each summand is stable under the action of $D$. The condition $\left(\mathbf{K}_{r}\right)$ just says that rank $\Lambda_{\varphi} / \mathcal{F}_{\varphi}=n r_{\varphi}$, in which case $\Lambda_{\varphi} / \mathcal{F}_{\varphi}$ is locally on $S$ isomorphic to the direct sum of $r_{\varphi}$ copies of the simple representation $F^{n} \otimes_{\varphi} R$ of $D \otimes_{F, \varphi} R \simeq \mathrm{M}_{n}(R)$. On the summand $\Lambda_{\varphi} / \mathcal{F}_{\varphi}, \iota(\pi)$ acts as $\varphi(\pi) \operatorname{Id}_{\mathcal{F}_{\varphi}}$. Let $\psi=\psi_{0}$. It then follows that $Q_{A_{\psi_{0}}}(\iota(\pi))$ annihilates all summands $\Lambda_{\varphi} / \mathcal{F}_{\varphi}$, for those $\varphi$ with $\varphi_{\left.\right|_{F t}}=\psi_{0}$ and $\varphi \neq \varphi_{0}$, and $Q_{A_{\psi_{0}}}(\iota(\pi))$ induces an isomorphism on $\Lambda_{\varphi_{0}} / \mathcal{F}_{\varphi_{0}}$, which implies the second Eisenstein condition. The first and third Eisenstein conditions are proved in an analogous way.

For $\varphi$ with $\varphi_{\left.\right|_{F t}} \neq \psi_{0}$, the subspace $\mathcal{F}_{\varphi}$ is trivial, i.e., either equal to (0) or to $\Lambda_{\varphi}$. On the other hand, using Morita equivalence, the $\mathrm{M}_{n}(R)$ stable summand $\mathcal{F}_{\varphi_{0}}$ of $\Lambda_{\varphi_{0}}$ corresponds to a hyperplane of $F^{n} \otimes_{F, \varphi_{0}} R$. It now follows that the geometric generic fiber of $\mathbb{M}_{r}$ is isomorphic to the projective space of hyperplanes in $\overline{\mathbb{Q}}_{p}^{n}$, i.e., to $\mathbb{P}^{n-1}$ (Grothendieck's convention).

The geometric special fiber $\overline{\mathbb{M}}_{r}=\mathbb{M}_{r} \otimes_{O_{E}} \bar{k}$ can be described as follows. Let $W_{\psi}=\Lambda \otimes_{O_{F}, \psi} \bar{k}$, an $e n^{2}$-dimensional vector space with its endomorphism $\Pi=\iota(\Pi)$. Let $S=\operatorname{Spec} R$, for a $\bar{k}$-algebra $R$, and let $\left(\mathcal{F}_{\psi}\right)_{\psi \in \Psi}$ be a point in $\overline{\mathbb{M}}_{r}(S)$. Let first $\psi \neq \psi_{0}$. By the third Eisenstein condition, 
$\mathcal{F}_{\psi}$ is a direct summand of rank $\left(e-a_{\psi}\right) n^{2}$ containing the image of $\Pi^{a_{\psi} n}$. Since these two submodules are direct summands of the same rank, they are equal.

Now let $\psi=\psi_{0}$, and set $W_{0}=W_{\psi_{0}}$ and $a_{0}=a_{\psi_{0}}$. Then, due to the action of $O_{\tilde{F}}$, we obtain a $\mathbb{Z} / n$-grading

$$
W_{0}=\bigoplus_{k \in \mathbb{Z} / n} W_{0, k}
$$

and $\Pi$ is an endomorphism of degree one. Forgetting the subspaces $\mathcal{F}_{\psi}$ with $\psi \neq \psi_{0}$, we have an identification

$$
\begin{aligned}
\overline{\mathbb{M}}_{r}(S)=\left\{\mathcal{F}_{0} \subset W_{0} \otimes_{\bar{k}} \mathcal{O}_{S}\right. & \mid \Pi \text {-stable graded direct summand, } \\
& \operatorname{rank}\left(W_{0, k, S} / \mathcal{F}_{0, k}\right)=a_{0} n+1, \forall k \in \mathbb{Z} / n, \\
& \text { and } \left.\left.\left.1^{\prime}\right) \text { and } 2^{\prime}\right)\right\} .
\end{aligned}
$$

Here we have set $W_{0, S}=W_{0} \otimes_{\bar{k}} \mathcal{O}_{S}$ and $W_{0, k, S}=W_{0, k} \otimes_{\bar{k}} \mathcal{O}_{S}$, and $\left.1^{\prime}\right)$ and $2^{\prime}$ ) are as follows:

$$
\begin{aligned}
& \left.1^{\prime}\right) \quad \Pi^{\left(a_{0}+1\right) n} \mid\left(W_{0, S} / \mathcal{F}_{0}\right) \quad=0 \\
& \left.2^{\prime}\right) \bigwedge^{n+1}\left(\Pi^{a_{0} n} \mid\left(W_{0, S} / \mathcal{F}_{0}\right)\right)=0 .
\end{aligned}
$$

Of course, we have used here (3.22).

Let us now apply Lemma 4.10 to the $\mathcal{O}_{S}$-dual $W_{0, S}^{*}$, its endomorphism $f$ induced by $\left(\Pi^{*}\right)^{a_{0} n}$ and its submodule $V=\left(W_{0, S} / \mathcal{F}_{0}\right)^{*}$, in which case $\operatorname{rank} W_{0, S}^{*}=e n^{2}$, and $\operatorname{rank} V=\left(a_{0} n+1\right) n$, and $r=a_{0} n^{2}$, and $s=n$. We conclude that $\operatorname{Ker}\left(\Pi^{*}\right)^{a_{0} n} \otimes_{\bar{k}} \mathcal{O}_{S} \subset V$. Translated back into $\mathcal{F}_{0}$, we obtain a chain of inclusions of direct summands of $W_{0, S}$,

$$
\operatorname{Im}\left(\Pi^{\left(a_{0}+1\right) n}\right) \otimes_{\bar{k}} \mathcal{O}_{S} \subset \mathcal{F}_{0} \subset \operatorname{Im}\left(\Pi^{a_{0} n}\right) \otimes_{\bar{k}} \mathcal{O}_{S} .
$$

In the Drinfeld case $r=r^{\circ}$ we have $a_{0}=0$. Let us write $\mathbb{M}^{\circ}$ for $\mathbb{M}_{r^{\circ}}$.

Let us identify $\operatorname{Im} \Pi^{\left(a_{0}+1\right) n} / \operatorname{Im} \Pi^{a_{0} n}$ with $W_{0} / \Pi W_{0}$. Associating now to an $S$-valued point $\mathcal{F}_{0}$ of $\overline{\mathbb{M}}_{r}$ the locally direct summand

$$
\mathcal{F}_{0} / \operatorname{Im}\left(\Pi^{\left(a_{0}+1\right) n}\right) \otimes_{\bar{k}} \mathcal{O}_{S} \subset\left(\operatorname{Im} \Pi^{a_{0} n} / \operatorname{Im} \Pi^{\left(a_{0}+1\right) n}\right) \otimes_{\bar{k}} \mathcal{O}_{S}=\left(\Lambda \otimes_{O_{F}, \varphi_{0}} \bar{k}\right) \otimes_{\bar{k}} \mathcal{O}_{S},
$$

we have obtained an $S$-valued point of the local model $\mathbb{M}^{\circ}$, more precisely an $S$-valued point of $\mathbb{M}^{0} \otimes_{O_{F}, \varphi_{0}} O_{E} \otimes_{O_{E}} \bar{k}$. Letting $S$ vary, this induces obviously an isomorphism of schemes over $\bar{k}$, 


$$
\mathbb{M}_{r} \otimes_{O_{E}} \bar{k} \simeq \mathbb{M}^{\circ} \otimes_{O_{F}} \bar{k}
$$

Therefore we obtain from the Drinfeld case:

Corollary 5.2. The geometric special fiber $\mathbb{M}_{r} \otimes_{O_{E}} \bar{k}$ is a reduced scheme, which has $n$ irreducible components, all of which have dimension $n-1$. Furthermore, the local rings of closed points of $\mathbb{M}_{r} \otimes_{O_{E}} \bar{k}$ are isomorphic to localizations in closed points of the $\bar{k}$-algebra $\bar{k}\left[X_{1}, \ldots, X_{n}\right] /\left(X_{1} \ldots . X_{n}\right)$.

Remark 5.3. We point out that $\mathbb{M}^{\circ}$ coincides with the standard local model for the triple $(G,\{\mu\}, K)$ consisting of $\mathrm{GL}_{n}$ and $\mu_{\left(1,0^{(n-1)}\right)}$ and the Iwahori subgroup, after extension of scalars to $\operatorname{Spec} O_{\breve{E}}$, cf. [7], comp. also [17, 18].

Corollary 5.4. $\mathbb{M}_{r}$ is flat over $O_{E}$.

Proof. Since the special fiber is reduced, it suffices by [8], Prop. 14.16 to show that a generic point of an irreducible component of the special fiber is in the closure of the general fibre. Since the general fiber and the special fibre of $\mathbb{M}_{r}$ have the same dimension $n-1$ and since $\mathbb{M}_{r}$ is proper it follows that at least one irreducible component of the special fiber is contained in the closure of the generic fiber. The claim therefore follows from the following lemma.

Lemma 5.5. There is an action of $\mathbb{Z} / n$ on $\mathbb{M}_{r} \otimes_{O_{E}} O_{\breve{E}}$, which induces a transitive action on the set of irreducible components of $\mathbb{M}_{r} \otimes_{O_{E}} \bar{k}$.

Proof. The action is given by sending $\mathcal{F}=\oplus_{\tilde{\psi} \in \tilde{\Psi}} \mathcal{F}_{\tilde{\psi}}$ to $\mathcal{F}^{\prime}$ with

$$
\left(\mathcal{F}^{\prime}\right)_{\tilde{\psi}}=\mathcal{F}_{\tilde{\psi} \tau}, \quad \tilde{\psi} \in \tilde{\Psi} .
$$

Here $\tau$ is taken from the presentation of $O_{D}$ in (3.3). Indeed, since the $O_{F}$-structure of $\left(\Lambda \otimes_{\mathbb{Z}_{p}} \mathcal{O}_{S}\right) / \mathcal{F}^{\prime}$ coincides with that of $\left(\Lambda \otimes_{\mathbb{Z}_{p}} \mathcal{O}_{S}\right) / \mathcal{F}$, the Eisenstein conditions are satisfied for $\left(\Lambda \otimes_{\mathbb{Z}_{p}} \mathcal{O}_{S}\right) / \mathcal{F}^{\prime}$, since they are satisfied for $\left(\Lambda \otimes_{\mathbb{Z}_{p}} \mathcal{O}_{S}\right) / \mathcal{F}$. On the other hand, for any $\psi \in \Psi$,

$$
\operatorname{rank}\left(\mathcal{F}^{\prime}\right)_{\psi}=\sum_{\tilde{\psi} \in \tilde{\Psi}_{\psi}} \operatorname{rank}\left(\mathcal{F}^{\prime}\right)_{\tilde{\psi}}=\sum_{\tilde{\psi} \in \tilde{\Psi}_{\psi}} \operatorname{rank} \mathcal{F}_{\tilde{\psi} \tau}=\sum_{\tilde{\psi} \in \tilde{\Psi}_{\psi}} \operatorname{rank} \mathcal{F}_{\tilde{\psi}}=r_{\psi} .
$$

Therefore, $\mathcal{F}^{\prime}$ also satisfies the condition $\left(\mathbf{R}_{r}\right)$.

That the action of $\mathbb{Z} / n$ on the set of irreducible components of $\mathbb{M}_{r} \otimes_{O_{E}} \bar{k}$ is transitive, follows from the corresponding fact for $\mathbb{M}^{\circ}$ (the Drinfeld case) (the isomorphism (5.4) is obviously equivariant for the action of $\mathbb{Z} / n$ ).

Corollary 5.6. The scheme $\mathbb{M}_{r}$ is normal. 
Proof. Indeed, $\mathbb{M}_{r}$ is flat over $O_{E}$, with normal generic fiber (even regular, cf. Lemma 5.1), and reduced special fiber. These properties imply that $\mathbb{M}_{r}$ is normal, cf. [19], Prop. 9.2.

Remark 5.7. If the Conjecture 2.6 were true, it would follow that $\mathbb{M}_{r}$ has semi-stable reduction, in particular $\mathbb{M}_{r}$ would be regular. However, we are unable to prove these stronger assertions.

Let $\mathcal{M}_{r}^{\prime}$ be the closed formal subscheme of $\mathcal{M}_{r}$ which is given by the Kottwitz condition $\left(\mathbf{K}_{r}\right)$. By Corollary 4.7 the special fibers of $\mathcal{M}_{r}$ and $\mathcal{M}_{r}^{\prime}$ are identical.

Proposition 5.8. The two formal schemes $\mathcal{M}_{r}$ and $\mathcal{M}_{r}^{\prime}$ are identical. Both are $p$-adic and flat over Spf $O_{\breve{E}}$, with special fiber $\mathcal{M}_{r} \times_{\operatorname{Spf} O_{\breve{E}}} \operatorname{Spec} \bar{k}$ a reduced scheme. All their completed local rings are normal.

Proof. We use the local model diagram

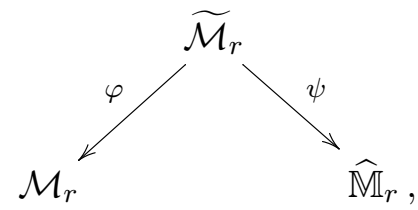

where $\widehat{\mathbb{M}}_{r}$ denotes the formal completion of $\mathbb{M}_{r} \times_{\text {Spec } O_{E}}$ Spec $O_{\breve{E}}$ along its special fiber. Here $\widetilde{\mathcal{M}}_{r}$ and the morphism $\varphi$ is obtained from $\mathcal{M}_{r}$ by adding to $(X, \iota, \rho)$ an $O_{D} \otimes_{\mathbb{Z}_{p}} \mathcal{O}_{S}$-linear isomorphism with the value at $S$ of the covariant crystal associated to $X$,

$$
\alpha: \Lambda \otimes_{\mathbb{Z}_{p}} \mathcal{O}_{S} \longrightarrow \mathbb{D}(X)_{S}
$$

The morphism $\psi$ maps the $S$-valued point $(X, \iota, \rho, \alpha)$ of $\widetilde{\mathcal{M}}_{r}$ to the submodule $\alpha^{-1}(\operatorname{Ker}(\mathbb{D}(X) \rightarrow$ Lie $X))$ of $\Lambda \otimes_{\mathbb{Z}_{p}} \mathcal{O}_{S}$. The theory of local models [21] tells us that the completed local ring of a point $x \in \mathcal{M}_{r}$ is isomorphic to the completed local ring of $\psi(\tilde{x})$, where $\tilde{x}$ is any point of $\widetilde{\mathcal{M}}_{r}$ mapping under $\varphi$ to $x$. Hence all completed local rings of points of $\mathcal{M}_{r}$ are isomorphic to completed rings of points of $\mathbb{M}_{r} \times_{\operatorname{Spec} O_{E}} \operatorname{Spec} O_{\breve{E}}$. Hence by Corollaries 5.4 and 5.2, the formal scheme $\mathcal{M}_{r}$ is flat over $\operatorname{Spf} O_{\breve{E}}$ with all completed local rings normal. That $\mathcal{M}_{r} \times \operatorname{Spf} O_{\breve{E}} \operatorname{Spec} \bar{k}$ is a reduced scheme follows from Corollary 4.15. Now the equality of $\mathcal{M}_{r}^{\prime}$ and $\mathcal{M}_{r}$ follows from Proposition 4.5 .

Corollary 5.9. The definition of $\mathcal{M}_{r}$ is independent of the choice of the uniformizer $\pi$ of $O_{F}$. 
Proof. Consider the formal scheme $\mathcal{N}_{r}$ that represents the moduli problem where the Kottwitz condition $\left(\mathbf{K}_{r}\right)$ is imposed but the Eisenstein conditions $\left(\mathbf{E}_{r}\right)$ are dropped. Let $\tilde{\pi}$ be another uniformizer, and let $\tilde{\mathcal{M}}_{r}$ be the corresponding formal scheme defined using the Eisenstein condition for $\tilde{\pi}$ instead of $\pi$. What has to be shown is that the formal subschemes $\mathcal{M}_{r}$ and $\tilde{\mathcal{M}}_{r}$ of $\mathcal{N}$ are identical. Let $\mathbb{N}_{r}$ be the local model corresponding to $\mathcal{N}_{r}$; then the local models $\mathbb{M}_{r}$ and $\tilde{\mathbb{M}}_{r}$ of $\mathcal{M}_{r}$ and $\tilde{\mathcal{M}}_{r}$ are closed subschemes of $\mathbb{N}_{r}$. It suffices to prove that $\mathbb{M}_{r}=\tilde{\mathbb{M}}_{r}$. But by Lemma 5.1 the generic fibers of $\mathbb{N}_{r}, \mathbb{M}_{r}$ and $\tilde{\mathbb{M}}_{r}$ all coincide and, by Corollary 5.4, $\mathbb{M}_{r}$ and $\tilde{\mathbb{M}}_{r}$ are equal to the flat closure of the generic fiber inside $\mathbb{N}_{r}$.

\section{The generic fiber (after Scholze)}

In this section, we prove the last point in Theorem 2.8, in the following form. For convenience, we introduce for a function $r: \varphi \mapsto r_{\varphi}$ the formal scheme $\tilde{\mathcal{M}}_{r}$ over $\operatorname{Spf} O_{\breve{E}}$ that represents the same moduli problem as $\mathcal{M}_{r}$, except that we drop the condition that the height of $\rho$ be zero. Then our original formal scheme $\mathcal{M}_{r}$ is an open and closed formal subscheme of $\tilde{\mathcal{M}}_{r}$.

Let $r^{\circ}$ be the Drinfeld function, i. e., $r_{\varphi}^{\circ}=0, \forall \varphi \neq \varphi_{0}$. We write $\tilde{\mathcal{M}}^{\circ}=$ $\tilde{\mathcal{M}}_{r^{\circ}}$.

We will prove the following theorem. We use the embedding $\breve{F} \hookrightarrow \breve{E}$ defined by the natural map $\breve{F}=F \otimes_{F^{t}} \breve{\mathbb{Q}}_{p} \stackrel{\varphi_{0} \otimes \text { id }}{\longrightarrow} E \otimes_{E^{t}} \breve{\mathbb{Q}}_{p}=\breve{E}$.

Theorem 6.1. There is an isomorphism of adic spaces over $\operatorname{Spa}\left(\breve{E}, O_{\breve{E}}\right)$,

$$
\left(\tilde{\mathcal{M}}_{r}\right)^{a d} \simeq\left(\tilde{\mathcal{M}}^{0} \hat{\otimes}_{O_{\breve{F}}} O_{\breve{E}}\right)^{a d}
$$

This theorem implies the last point in Theorem 2.8. Indeed, passing to the open and closed sublocus where the universal quasi-isogeny $\varrho$ has height zero, we obtain a similar isomorphism when $\tilde{\mathcal{M}}_{r}$ is replaced by $\mathcal{M}_{r}$ and $\tilde{\mathcal{M}}^{\circ}$ by $\mathcal{M}^{\circ}$ (the proof of Theorem 6.1 will show that the isomorphism in question is compatible with the decompositions according to the height). Since by Drinfeld's theorem $\left(\mathcal{M}^{\circ}\right)^{a d} \simeq \Omega_{F}^{n}$, we deduce the desired isomorphism

$$
\left(\mathcal{M}_{r}\right)^{a d} \simeq \hat{\Omega}_{F} \otimes_{F} \breve{E}=\left(\hat{\Omega}_{F} \otimes_{O_{F}} O_{\breve{E}}\right)^{a d} .
$$

In the proof of Theorem 6.1, we will use the following notation. We denote by $\left(\mathbb{X}, \iota_{\mathbb{X}}\right)$ the framing object for the moduli problem $\tilde{\mathcal{M}}_{r}$. Let $M(\mathbb{X})_{\mathbb{Q}_{p}}$ be its rational Diendonné module.

Let $V$ be a free $D$-module of rank one, and let $\Lambda$ be an $O_{D}$-lattice in $V$. Let $G=\mathrm{GL}_{D}(V)$, considered as a linear algebraic group over $\mathbb{Q}_{p}$. The 
function $r$ defines a $G$-homogeneous projective variety $\mathcal{F}$ over $E$. If $R$ is a $\overline{\mathbb{Q}}_{p}$-algebra, then $\mathcal{F}(R)$ parametrizes $D$-linear surjective homomorphisms into locally free $R$-modules

$$
V \otimes_{\mathbb{Q}_{p}} R \longrightarrow \mathcal{F}
$$

such that under the decomposition $\mathcal{F}=\bigoplus_{\varphi} \mathcal{F}_{\varphi}$, we have $\operatorname{rank}\left(\mathcal{F}_{\varphi}\right)=$ $r_{\varphi} n$. In other words, $\mathcal{F}$ coincides with the generic fiber of the local model, $\mathbb{M}_{r} \times_{\operatorname{Spec}} O_{E} \operatorname{Spec} E$, cf. Lemma 5.1.

Let $K_{0} \subset G\left(\mathbb{Q}_{p}\right)$ be the stabilizer of the lattice $\Lambda$. For any open subgroup $K \subset K_{0}$, we obtain the corresponding member $\mathbb{M}_{K}$ of the RZ-tower over $\left(\tilde{\mathcal{M}}_{r}\right)^{a d}$. These coverings of $\left(\tilde{\mathcal{M}}_{r}\right)^{a d}$ parametrize level- $K$-structures on the universal object $X / \tilde{\mathcal{M}}_{r}$,

$$
\alpha: \Lambda \longrightarrow T(X) \bmod K .
$$

We denote by

$$
\pi_{K}: \mathbb{M}_{K} \longrightarrow\left(\mathcal{F} \otimes_{E} \breve{E}\right)^{a d}
$$

the crystalline period maps, which are compatible with changes in $K$.

In [24] Scholze and Weinstein define a preperfectoid space $\mathbb{M}_{\infty}$ by imposing a full level structure on $T(X)$. In particular, there is a morphism

$$
\mathbb{M}_{\infty} \longrightarrow \underbrace{}_{\lim } \mathbb{M}_{K}
$$

which induces a bijection for any algebraically closed extension $C$ of $\overline{\mathbb{Q}}_{p}$ which is complete for a $p$-adic valuation,

$$
\mathbb{M}_{\infty}(C) \simeq \lim _{\longleftarrow} \mathbb{M}_{K}(C) .
$$

We denote by $\pi_{\infty}: \mathbb{M}_{\infty} \longrightarrow\left(\mathcal{F} \otimes_{E} \breve{E}\right)^{a d}$ the induced period mapping. In [24], the following description of $\mathbb{M}_{\infty}(C)$ is given. Let $\mathcal{O}_{C}$ be the ring of integers in $C$.

Let $B_{\text {cris }}^{+}=A_{\text {cris }}\left(\mathcal{O}_{C} / p\right) \otimes_{\mathbb{Z}_{p}} \mathbb{Q}_{p}$ be Fontaine's ring attached to $C$. The Fargues-Fontaine curve ${ }^{4}$ is defined as $Y=\operatorname{Proj} P$, where $P$ is the graded ring

$$
P=\bigoplus_{d \geq 0}\left(B_{\text {cris }}^{+}\right)^{\phi=p^{d}} .
$$

\footnotetext{
${ }^{4}$ The Fargues-Fontaine curve is usually denoted by $X$; since this notation is already in use for the universal $p$-divisible group, we use the notation $Y$ instead.
} 
Then $Y$ is a connected separated regular noetherian scheme of dimension 1, equipped with the point $\infty \in Y$ corresponding to Fontaine's homomorphism

$$
\theta: B_{\text {cris }}^{+} \longrightarrow C \text {. }
$$

In [24] appears a description of $p$-divisible groups $X$ over $\mathcal{O}_{C}$, in terms of two vector bundles, $\mathcal{E}$ and $\mathcal{F}$. Here

- $\mathcal{F}=T \otimes_{\mathbb{Z}_{p}} \mathcal{O}_{Y}$

- $\mathcal{E}$ corresponds to the graded $P$-module $\bigoplus_{d \geq 0}\left(M_{\mathbb{Q}_{p}}\right)^{\phi=p^{d+1}}$.

Here $T=T(X)$ denotes the Tate module of the generic fibre of $X$, and $M_{\mathbb{Q}_{p}}=M(X)_{\mathbb{Q}_{p}}$ the rational Dieudonné module of the reduction modulo $p$ of $X$.

We now apply this description to the fibers of the universal $p$-divisible group $X$ at points of $\mathbb{M}_{\infty}(C)$, noting that the universal full level structure induces an isomorphism $T(X)=\Lambda$, and the universal quasi-isogeny an isomorphism of rational Dieudonné modules $M(X)_{\mathbb{Q}_{p}}=M(\mathbb{X})_{\mathbb{Q}_{p}} \otimes_{\mathbb{Q}_{p}} B_{\text {cris }}^{+}$. Accordingly we set $\mathcal{F}=\Lambda \otimes_{\mathbb{Z}_{p}} \mathcal{O}_{Y}$, and let $\mathcal{E}$ correspond to the graded module

$$
\left(\bigoplus_{d \geq 0} M(\mathbb{X})_{\mathbb{Q}_{p}} \otimes_{\breve{\mathbb{Q}}_{p}} B_{\text {cris }}^{+}\right)^{\phi=p^{d+1}} .
$$

We also fix a $D$-linear isomorphism $M(\mathbb{X})_{\mathbb{Q}_{p}}=V \otimes \mathbb{Q}_{p} \breve{\mathbb{Q}}_{p}$. The ScholzeWeinstein description therefore implies the following fact, cf. [24], Cor. 6.3.10 (and its extension to the EL-case in Thm. 6.5.4).

Theorem 6.2. There is an identification of $\mathbb{M}_{\infty}(C)$ with the set of injective $D$-linear homomorphisms of vector bundles on $Y$,

$$
f: \mathcal{F} \longrightarrow \mathcal{E}
$$

such that $\operatorname{supp}(\operatorname{Coker} f)=\{\infty\}$ and such that $\mathfrak{m}_{Y, \infty}$ kills $\operatorname{Coker} f$ and such that the induced surjective map

$$
\mathcal{E} \otimes_{\mathcal{O}_{X, \infty}} C=M(\mathbb{X})_{\mathbb{Q}_{p}} \otimes_{\breve{Q}_{p}} C=V \otimes_{\mathbb{Q}_{p}} C \stackrel{\varphi_{f}}{\longrightarrow} \text { Coker } f
$$

defines a point in $\mathcal{F}(C)$. Furthermore, the period morphism $\pi_{\infty}$ sends the point corresponding to $f$ to $\left[V \otimes_{\mathbb{Q}_{p}} C \stackrel{\varphi_{f}}{\longrightarrow}\right.$ Coker $\left.f\right]$.

It will be convenient to reformulate this last description. Let $Y_{F}=$ $Y \times_{\operatorname{Spec} \mathbb{Q}_{p}} \operatorname{Spec} F$. This is a finite étale cover $\psi: Y_{F} \longrightarrow Y$ of degree $d$, 
and the fiber $\psi^{-1}(\{\infty\})$ can be identified with $\left\{\infty_{\varphi} \mid \varphi: F \longrightarrow \overline{\mathbb{Q}}_{p}\right\}$. Since the vector bundles $\mathcal{F}$ and $\mathcal{E}$ are equipped with $F$-actions, they are of the form $\mathcal{F}=\psi_{*}\left(\mathcal{F}_{F}\right)$ and $\mathcal{E}=\psi_{*}\left(\mathcal{E}_{F}\right)$, with vector bundles $\mathcal{F}_{F}$ and $\mathcal{E}_{F}$ over $Y_{F}$.

Corollary 6.3. There is a natural identification of $\mathbb{M}_{\infty}(C)$ with the set of $D$-linear injective morphisms of vector bundles on $Y_{F}$,

$$
f_{F}: \mathcal{F}_{F} \longrightarrow \mathcal{E}_{F},
$$

such that supp Coker $f_{F} \subset \psi^{-1}(\{\infty\})$, with Coker $f_{F}$ killed by $\mathfrak{m}_{Y_{F}, \infty_{\varphi}} \forall \varphi$, and such that

$$
\operatorname{dim}_{C}\left(\operatorname{Coker} f_{F}\right)_{\infty_{\varphi}}=r_{\varphi} \cdot n, \forall \varphi .
$$

Furthermore, the period morphism $\pi_{\infty}$ sends the point corresponding to $f_{F}$ to the family of surjections $\left[\mathcal{E}_{F} \otimes_{Y_{F}, \infty_{\varphi}} C=V \otimes_{F, \varphi} C \longrightarrow\left(\operatorname{Coker} f_{F}\right)_{\infty_{\varphi}}\right]$, considered as a point in $\mathcal{F}(C)$.

Now we compare the previous descriptions for the given function $r$, and for the Drinfeld function $r^{\circ}$. Let $\mathbb{X}^{\circ}$ denote the framing object for $\tilde{\mathcal{M}}_{r^{\circ}}$. Then we may choose $D$-linear isomorphisms

$$
V \otimes_{\mathbb{Q}_{p}} \breve{\mathbb{Q}}_{p}=M\left(\mathbb{X}^{\circ}\right)_{\mathbb{Q}_{p}}=V \otimes_{\mathbb{Q}_{p}} \breve{\mathbb{Q}}_{p}=M(\mathbb{X})_{\mathbb{Q}_{p}}
$$

such that for the respective Frobenius endomorphisms

$$
F=\pi^{m} \cdot F^{\circ},
$$

where

$$
m=\#\left\{\varphi \mid r_{\varphi}=n\right\} .
$$

For the corresponding vector bundles on $Y_{F}$, we get

$$
\mathcal{E}_{F}=\mathcal{E}_{F}^{\circ} \otimes \mathcal{O}_{X_{F}} \mathcal{L}^{m},
$$

where $\mathcal{L}$ is the line bundle on $Y_{F}$ corresponding to the graded module

$$
\bigoplus_{d \geq 0}\left(F \otimes_{\mathbb{Q}_{p}} B_{\text {cris }}^{+}\right)^{\phi=\pi p^{d}} .
$$

We have a natural identification

$$
\mathcal{F}_{F}=\Lambda \otimes_{O_{F}} \mathcal{O}_{Y_{F}}=\mathcal{F}_{F}^{\circ} .
$$

On the other hand, we have a natural identification

$$
\mathcal{F}=\mathcal{F}^{\circ} \otimes_{F} E .
$$


Here, for a $\overline{\mathbb{Q}}_{p}$-algebra $R$, a point $\left[V \otimes_{\mathbb{Q}_{p}} R \longrightarrow \mathcal{F}\right]=\left[\left\{V \otimes_{F, \varphi} R \longrightarrow \mathcal{F}_{\varphi} \mid \varphi\right\}\right]$ of $\mathcal{F}$ is sent to $\left[\left\{V \otimes_{F, \varphi} R \longrightarrow \mathcal{F}_{\varphi}^{\circ} \mid \varphi\right\}\right]$, with

$$
\mathcal{F}_{\varphi}^{\circ}= \begin{cases}\mathcal{F}_{\varphi_{0}} & \text { if } \varphi=\varphi_{0} \\ V \otimes_{F, \varphi} R & \text { if } \varphi \neq \varphi_{0} .\end{cases}
$$

The general case of an $E$-algebra $R$ follows by descent. We point out that, by Morita equivalence, $\mathcal{F}^{\circ} \cong \mathbb{P}_{F}^{n-1}$.

Lemma 6.4. For a given $\varphi$, there exists a global section

$$
L T_{\varphi} \in \Gamma\left(Y_{F}, \mathcal{L}\right)=\left(F \otimes \mathbb{Q}_{p} B_{\text {cris }}^{+}\right)^{\phi=\pi}
$$

such that $L T_{\varphi}$ vanishes to first order at $\infty_{\varphi}$ and is non-vanishing at all other points. Furthermore, $L T_{\varphi}$ is unique up to $F^{\times}$.

Proof. The homomorphism $L T_{\varphi}: \mathcal{O}_{Y_{F}} \longrightarrow \mathcal{L}$ corresponds in the ScholzeWeinstein description to the Lubin-Tate formal group corresponding to the triple $(F, \varphi, \pi)$.

Lemma 6.5. Let $i: \mathcal{G}_{F} \longrightarrow \mathcal{E}_{F}$ be the injection of vector bundles on $Y_{F}$,

$$
\mathcal{G}_{F}=\left\{x \in \mathcal{E}_{F} \mid x \equiv 0 \quad \bmod \infty_{\varphi}, \forall \varphi \text { with } r_{\varphi}=n\right\} .
$$

Then the map $x \longmapsto x \cdot \Pi_{\left\{\varphi \mid r_{\varphi}=n\right\}} L T_{\varphi}$ defines an isomorphism $\mathcal{E}_{F}^{\circ} \simeq \mathcal{G}_{F}$.

Proof. The map

$$
x \longmapsto x \prod_{\left\{\varphi \mid r_{\varphi}=n\right\}} L T_{\varphi}
$$

obviously identifies $\mathcal{E}_{F}^{\circ}$ with a subbundle of $\mathcal{G}_{F}$. Comparing the degrees of $\mathcal{E}_{F}^{\circ}$ and $\mathcal{G}$, the result follows. (There is a formalism of degrees of vector bundles on $Y$ resembling the usual theory over smooth projective curves.)

Proposition 6.6. Under the identification $\mathcal{E}_{F}^{\circ}=\mathcal{G}_{F}$ and $\mathcal{F}_{F}=\mathcal{F}_{F}^{\circ}$, the map sending $f_{F}^{\circ} \in \operatorname{Hom}\left(\mathcal{F}_{F}^{\circ}, \mathcal{E}_{F}^{\circ}\right)$ to $f_{F}=i \circ f_{F}^{\circ} \in \operatorname{Hom}\left(\mathcal{F}_{F}, \mathcal{E}_{F}\right)$ defines a bijection $\mathbb{M}_{\infty}^{\circ}(C)=\mathbb{M}_{\infty}(C)$ which commutes with the period map $\pi_{\infty}^{\circ}$, resp. $\pi_{\infty}$, to $\mathcal{F}^{\circ}(C)=\mathcal{F}(C)$.

Proof. It is clear that Coker $f_{F}^{\circ}$ has support in $\left\{\infty_{\varphi_{0}}\right\} \cup\left\{\infty_{\varphi} \mid r_{\varphi}=n\right\}$, and that for $\varphi$ with $r_{\varphi}=n$, one has that $\left(\operatorname{Coker} f_{F}^{\circ}\right)_{\infty_{F}}=\mathcal{E}_{F} \otimes_{\mathcal{O}_{Y_{F}}, \infty_{\varphi}} C$. Conversely, any $f_{F} \in \mathbb{M}_{\infty}(C)$ has to factor through $\mathcal{G}_{F}$ and has the correct cokernel at $\infty_{\varphi_{0}}$. The assertion regarding the period map is obvious from the way that $\mathcal{F}(C)$ is identified with $\mathcal{F}^{\circ}(C)$. 
Corollary 6.7. Under the identification $\mathcal{F}^{a d}=\left(\mathcal{F}^{\circ} \otimes_{F} E\right)^{\text {ad }}$, the images of the period morphisms $\pi_{\infty}$ and $\pi_{\infty}^{\circ} \otimes_{\breve{F}} \breve{E}$, coincide.

Proof. All maps are partially proper [6], hence it suffices to prove for all algebraically closed complete extensions $C$ of $\overline{\mathbb{Q}}_{p}$ that

$$
\operatorname{Im} \pi_{\infty}(C)=\operatorname{Im} \pi_{\infty}^{\circ}(C) .
$$

This follows from the previous proposition.

Proof of Theorem 6.1. We need to construct an isomorphism

$$
\mathbb{M}_{K_{0}} \simeq \mathbb{M}_{K_{0}}^{\circ} \otimes_{\breve{F}} \breve{E}
$$

It suffices to construct the isomorphism on the open and closed subloci $\mathbb{M}_{K_{0}}^{(n)}$, resp. $\mathbb{M}_{K_{0}}^{\circ(n)}$, where the height of $\varrho$ is a fixed integer $n$. But the fibers of the period morphisms $\pi_{K_{0}}$, resp. $\pi_{K_{0}}^{\circ}$, through $\mathbb{M}_{K_{0}}^{(n)}$, resp. $\mathbb{M}_{K_{0}}^{\circ(n)}$, can both be identified with $G\left(\mathbb{Q}_{p}\right)^{\circ} / K_{0}$, where under the identification $G\left(\mathbb{Q}_{p}\right)=D^{\times}$, we have

$$
G\left(\mathbb{Q}_{p}\right)^{\circ}=\left\{x \in D^{\times} \mid \text {ord } \operatorname{det} x=0\right\} .
$$

Since $K_{0}=G\left(\mathbb{Q}_{p}\right)^{\circ}$, the period maps identify $\mathbb{M}_{K_{0}}^{(n)}$ and $\mathbb{M}_{K_{0}}^{\circ(n)}$ with open adic subsets of $\left(\mathcal{F} \otimes_{E} \breve{E}\right)^{a d}=\left(\mathcal{F}^{\circ} \otimes_{F} \breve{E}\right)^{a d}$. The assertion therefore follows from Corollary 6.7.

\section{The unramified case}

In this section we prove Theorem 2.7. Hence in this section $F / \mathbb{Q}_{p}$ is unramified. Since we fixed an embedding $\varphi_{0}: F \longrightarrow \overline{\mathbb{Q}}_{p}$, we may identify

$$
\operatorname{Hom}_{\mathbb{Q}_{p}}\left(F, \overline{\mathbb{Q}}_{p}\right)=\left\{\sigma^{i} \mid i \in \mathbb{Z} / d\right\} .
$$

In particular, $E=F$, via $\varphi_{0}$. We abbreviate

$$
r_{i}=r_{\varphi_{0} \circ \sigma^{i}}, \quad i \in \mathbb{Z} / d
$$

Let $S$ be a $O_{F}$-scheme, such that $p$ is nilpotent on $S$. We have a decomposition

$$
O_{F} \otimes_{\mathbb{Z}_{p}} \mathcal{O}_{S}=\bigoplus_{i \in \mathbb{Z} / d} \mathcal{O}_{S},
$$

where the action of $O_{F}$ on the $i$-th summand is via $\sigma^{i}$. If $(X, \iota)$ is a formal $O_{D}$-module over $S$, we correspondingly obtain a decomposition, 


$$
\operatorname{Lie} X=\bigoplus_{i \in \mathbb{Z} / d} \operatorname{Lie}_{i} X
$$

where $t \in \operatorname{Lie}_{i} X$ iff $\iota(x)(t)=\sigma^{i}(x) t, \forall x \in O_{F}$.

Lemma 7.1. The condition $\left(\mathbf{R}_{r}\right)$ implies the conditions $\left(\mathbf{K}_{r}\right)$ and $\left(\mathbf{E}_{r}\right)$.

Proof. For the implication $\left(\mathbf{R}_{r}\right) \Rightarrow\left(\mathbf{E}_{r}\right)$, we refer to Remarks 3.9, (iii). The condition $\left(\mathbf{D}_{r}\right)$ implies $\left(\mathbf{K}_{r}\right)$ by Proposition 5.8.

We will now consider the display of $X$. To simplify the notation, we assume that $S=\operatorname{Spec} R$, where $R$ is an $O_{F}$-algebra. Let $W\left(O_{F}\right)$ be the ring of Witt vectors of $O_{F}$, and define a ring homomorphism by

$$
\lambda: O_{F} \longrightarrow W\left(O_{F}\right), \quad w_{m}(\lambda(x))=\sigma^{m}(x) .
$$

Then $\lambda$ is Frobenius equivariant, i.e., $\lambda(\sigma(x))={ }^{F}(\lambda(x))$. For a $O_{F}$-algebra $R$, we obtain

$$
\bar{\lambda}: O_{F} \longrightarrow W\left(O_{F}\right) \longrightarrow W(R) .
$$

Let

$$
\bar{\lambda}^{(i)}=\bar{\lambda} \circ \sigma^{i}={ }^{F^{i}} \circ \bar{\lambda}: O_{F} \longrightarrow W(R) .
$$

Let $\mathcal{P}=(P, Q, F, \dot{F})$ be the display of $X$ over $R$. Hence $P$ is a finitely generated projective $W(R)$-module, and $Q$ is submodule of $P$, and

$$
F: P \longrightarrow P, \dot{F}: Q \longrightarrow P .
$$

The action of $O_{F}$ on $\mathcal{P}$ defines decompositions,

$$
P=\bigoplus P_{i}, \quad Q=\bigoplus Q_{i},
$$

where $O_{F}$ acts on the $i$-th summand via $\bar{\lambda}^{(i)}$. The operators $F$ and $\dot{F}$ are of degree 1 with respect to this $\mathbb{Z} / d$-grading,

$$
F: P_{i} \longrightarrow P_{i+1}, \quad \dot{F}: Q_{i} \longrightarrow P_{i+1} .
$$

Then $\operatorname{rank} P_{i}=n^{2}, \forall i$, by Proposition 4.3. The rank condition says:

$$
\begin{gathered}
P_{0} / Q_{0} \text { is a locally free } R \text {-module of rank } n \\
P_{i}=Q_{i} \text { if } r_{i}=0, I_{R} \cdot P_{i}=Q_{i} \text { if } r_{i}=n .
\end{gathered}
$$

We choose a normal decomposition,

$$
P_{i}=T_{i} \oplus L_{i}, \text { for all } i .
$$


Then

$$
F \oplus \dot{F}: T_{i} \oplus L_{i} \longrightarrow P_{i+1}
$$

is a ${ }^{F}$-linear isomorphism, for all $i$. Let $i \neq 0$. When $r_{i}=0$, then $P_{i}=Q_{i}$ and we obtain an isomorphism

$$
\dot{F}^{\#}: W(R) \otimes_{F, W(R)} P_{i} \longrightarrow P_{i+1}
$$

When $r_{i}=n$, then $L_{i}=(0)$, and we obtain an isomorphism

$$
F^{\#}: W(R) \otimes_{F, W(R)} P_{i} \longrightarrow P_{i+1}
$$

We now define ${ }^{F^{d}}$-linear homomorphisms,

$$
F_{\mathrm{rel}}^{\#}: P_{0}^{\left(p^{d}\right)} \longrightarrow P_{0}, \dot{F}_{\mathrm{rel}}^{\#}: Q_{0}^{\left(p^{d}\right)} \longrightarrow P_{0}
$$

as compositions,

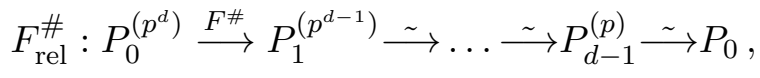

resp.

$$
\dot{F}_{\text {rel }}^{\#}: Q_{0}^{\left(p^{d}\right)} \longrightarrow P_{1}^{\left(p^{d-1}\right)} \stackrel{\sim}{\longrightarrow} \ldots \stackrel{\sim}{\longrightarrow} P_{d-1}^{(p)} \stackrel{\sim}{\longrightarrow} P_{0} .
$$

Here the isomorphisms in the last two lines are either (7.2) or (7.3). We note that $P_{0}$ is a finitely generated projective $W(R)$-module, that $Q_{0}$ is a submodule and $F_{\text {rel }}$ and $\dot{F}_{\text {rel }}$ satisfy the following relations,

$$
\begin{aligned}
& \dot{F}_{\text {rel }}\left({ }^{V} \xi x\right)={ }^{F^{d-1}} \xi \cdot F_{\text {rel }}(x), \quad x \in P_{0} . \\
& p \cdot \dot{F}_{\text {rel }}(y)=F_{\text {rel }}(y), \quad y \in Q_{0} \text {. }
\end{aligned}
$$

Indeed, the first identity reflects the $F^{d}$-linearity of $\dot{F}_{\text {rel }}$; the second identity comes from the fact that a similar identity holds for $\dot{F}$ and $F$. The quadruple $\left(P_{0}, Q_{0}, F_{\text {rel }}, \dot{F}_{\text {rel }}\right)$ is a $d$-display in the following sense:

Definition 7.2. Let $d \geq 1$ be a natural number. Let $R$ be a ring such that $p$ is a nilpotent in $R$. An $d$-display over $R$ is a quadruple $(P, Q, F, \dot{F})$, where $P$ is a finitely generated projective $W(R)$-module, $Q$ a submodule of $P$ and $F: P \rightarrow Q$ and $\dot{F}: Q \rightarrow P$ are ${ }^{F^{d}}$-linear maps such that the following properties are satisfied:

1. $I_{R} P \subset Q$, and $P / Q$ is a direct summand of the $R$-module $P / I_{R} P$. 
2. The linearization of $\dot{F}$,

$$
\dot{F}^{\sharp}: W(R) \otimes_{F^{d}, W(R)} Q \longrightarrow P
$$

is surjective.

3. For $x \in P$ and $w \in W(R)$,

$$
\dot{F}({ } w x)={ }^{F^{d-1}} w F(x) .
$$

We will now use the theory of relative Witt vectors and relative displays. We denote by $q=p^{d}$ the number of elements in the residue class field $\kappa$ of $O_{F}$. Also, when we use $O_{F}$ as a subscript, we simply write $O$. For an $O_{F^{-}}$ algebra $R$, we denote by $W_{O}(R)$ the ring of relative Witt vectors defined by the Witt polynomials

$$
w_{n}^{\prime}\left(x_{0}, \ldots, x_{n}\right)=x_{0}^{q^{n}}+p x_{1}^{q^{n-1}}+\cdots+p^{n} x_{n} .
$$

We have a canonical morphism $u: W(R) \rightarrow W_{O}(R)$ such that

$$
\begin{aligned}
& u\left({ }^{F^{d}} \xi\right)={ }^{F^{\prime}} u(\xi) \\
& u\left({ }^{V} \xi\right)={ }^{V^{\prime}}\left(u\left({ }^{F^{d-1}} \xi\right)\right),
\end{aligned}
$$

cf. [5], Prop. 1.2. Here we denoted by a prime the operators on $W_{O}(R)$.

We now show how to associate to $\left(P_{0}, Q_{0}, F_{\text {rel }}, \dot{F}_{\text {rel }}\right)$ a relative display $\left(P^{\prime}, Q^{\prime}, F^{\prime}, \dot{F}^{\prime}\right)$ with respect to $W_{O}(R)$ (replace the Witt vectors by the relative Witt vectors in the definition of a display).

We set

$$
\begin{aligned}
& P^{\prime}=W_{O}(R) \otimes_{u, W(R)} P_{0} \\
& Q^{\prime}=\operatorname{Ker}\left(W_{O}(R) \otimes_{u, W(R)} P_{0} \longrightarrow P_{0} / Q_{0}\right) .
\end{aligned}
$$

Here the last homomorphism is given by the composition

$$
\begin{aligned}
W_{O}(R) \otimes_{u, W(R)} P_{0} \longrightarrow & W_{O}(R) / I_{O}(R) \otimes_{u, W(R)} P_{0}=R \otimes_{u, W(R)} P_{0} \\
& =P_{0} / I(R) P_{0} \longrightarrow P_{0} / Q_{0} \\
F^{\prime} & ={ }^{F^{\prime}} \otimes F_{\text {rel }}: P^{\prime} \longrightarrow P^{\prime}
\end{aligned}
$$

Note that this makes sense because of (7.4), and defines a ${ }^{F^{d}}$-linear endomorphism of $P^{\prime}$. It remains to define $\dot{F}^{\prime}: Q^{\prime} \longrightarrow P^{\prime}$ with the following properties, 


$$
\begin{aligned}
\dot{F}^{\prime}\left({ }^{V^{\prime}} \xi x\right) & =\xi \cdot F^{\prime}(x), & & x \in P^{\prime} \\
\dot{F}^{\prime}(\xi \otimes y) & ={ }^{F^{\prime}} \xi \otimes \dot{F}_{\text {red }}(y), & & y \in Q .
\end{aligned}
$$

More precisely, consider the normal decomposition $P_{0}=T_{0} \oplus L_{0}$. Then

$$
Q^{\prime}=\left(I_{O}(R) \otimes_{W(R)} T_{0}\right) \oplus\left(W_{O}(R) \otimes_{W(R)} L_{0}\right) .
$$

We define $\dot{F}^{\prime}$ on the first, resp. second summand by

$$
\begin{aligned}
& \dot{F}^{\prime}\left(V^{\prime} \xi \otimes t_{0}\right)=\xi \otimes F_{\text {rel }}\left(t_{0}\right), \quad t_{0} \in T_{0} \\
& \dot{F}^{\prime}\left(\xi \otimes l_{0}\right)={ }^{F^{\prime}} \xi \otimes F_{\text {rel }}\left(l_{0}\right), \quad l_{0} \in L_{0} \text {. }
\end{aligned}
$$

Claim: The identities (7.5) are satisfied.

We start with the second identity. Let

$$
y={ }^{\prime} \eta \cdot t_{0}+l_{0} \quad, t_{0} \in T_{0}, l_{0} \in L_{0}
$$

For the second summand, the identity to be checked is the definition of $\dot{F}^{\prime}$. So we may take $l_{0}=0$. Now

$$
\begin{aligned}
\xi \otimes V^{\prime} \eta \cdot t_{0} & =\xi \cdot u\left({ }^{V^{\prime}} \eta\right) \otimes t_{0}=\xi \cdot{ }^{\prime} u\left({ }^{F^{d-1}} \eta\right) \otimes t_{0} \\
& ={ }^{V^{\prime}}\left({ }^{F^{\prime}} \xi \cdot u\left({ }^{F^{d-1}} \eta\right)\right) \otimes t_{0} .
\end{aligned}
$$

Hence the LHS of the identity to be checked is

$$
\begin{aligned}
\dot{F}^{\prime}\left(\xi \otimes V^{\prime} \eta t_{0}\right) & ={ }^{F^{\prime}} \xi u\left({ }^{F^{d-1}} \eta\right) \otimes F_{\text {rel }}\left(t_{0}\right) \\
& ={ }^{F^{\prime}} \xi \otimes{ }^{d-1} \eta \cdot F_{\text {rel }}\left(t_{0}\right) \\
& ={ }^{F^{\prime}} \xi \otimes \dot{F}_{\text {rel }}\left({ }^{\prime} \eta t_{0}\right)
\end{aligned}
$$

where in the last equation we used (7.4). The second identity of (7.5) is proved.

Now we check the first identity. It suffices to check that

$$
\dot{F}^{\prime}\left(V^{\prime} \xi \otimes x\right)=\xi \otimes F_{\text {rel }}(x), \quad x \in P_{0} .
$$

If $x=t_{0} \in T_{0}$, this holds by definition. Let $x=l_{0} \in L_{0}$. Then

$$
\begin{aligned}
\dot{F}^{\prime}\left(V^{\prime} \xi \otimes l_{0}\right) & =p \xi \otimes \dot{F}_{\text {rel }}\left(l_{0}\right)=\xi \otimes p \dot{F}_{\text {rel }}\left(l_{0}\right) \\
& =\xi \otimes F_{\text {rel }}\left(l_{0}\right)
\end{aligned}
$$

where in the last equation we used (7.4). 
We now have checked that $\left(P^{\prime}, Q^{\prime}, F^{\prime}, \dot{F}^{\prime}\right)$ is a relative display relative to $O_{F}$. By functoriality this relative display has an $O_{D}$-action. Its Lie algebra $P^{\prime} / Q^{\prime}$ coincides with $\operatorname{Lie}_{0} X=P_{0} / Q_{0}$. Therefore the action of $O_{D}$ on $P^{\prime} / Q^{\prime}$ is special in the sense of Drinfeld (satisfies condition $\left(\mathbf{R}_{r}\right.$ )), i.e., we are in the case of Proposition 3.6. This implies automatically that the relative display is nilpotent.

Let $R$ be an $O_{F}$-algebra where $\pi R$ is a nilpotent ideal. By a theorem of Ahsendorf [1], Thm. 5.3.8, there is an equivalence of categories between the category of $p$-divisible formal $O_{F}$-modules over $R$ and the category of nilpotent relative displays (this holds even without the hypothesis that $F / \mathbb{Q}_{p}$ is unramified). We therefore obtain a formal $O_{F}$-module $X^{\prime}$ over $S=\operatorname{Spec} R$, which is a special formal $O_{D}$-module because Lie $X^{\prime}=P^{\prime} / Q^{\prime}$.

Applying the above construction to the framing object $\left(\mathbb{X}, \iota_{\mathbb{X}}\right)$, we obtain a special formal $O_{D^{-m o d u l e}}\left(\mathbb{X}^{\prime}, \iota_{\mathbb{X}^{\prime}}\right)$ that we use as a framing object for the Drinfeld functor $\mathcal{M}_{r^{\circ}}$. Since the above construction is functorial in $S$, we obtain a morphism of formal schemes over $\operatorname{Spf} O_{\breve{F}}$,

$$
\mathcal{M}_{r} \longrightarrow \mathcal{M}_{r^{\circ}} .
$$

Theorem 7.3. The morphism (7.6) is an isomorphism. In particular, there is an isomorphism of formal schemes over $\operatorname{Spf} O_{\breve{F}}$,

$$
\mathcal{M}_{r} \simeq \hat{\Omega}_{F}^{n} \hat{\otimes}_{O_{F}} O_{\breve{F}} .
$$

Proof. Let $R$ be a $\kappa_{F}$-algebra. We have (compare (4.11)) described a functor $\mathcal{P} \mapsto \mathcal{P}^{\prime}$ which associates to the display of a formal $O_{D}$-module with condition $\left(\mathbf{D}_{r}\right)$ the display of a special formal $O_{D^{-}}$-module with condition $\left(\mathbf{D}_{r^{\circ}}\right)$. In the unramified case we take $\pi=p$. Since $a_{\psi}=0$ or 1 , we see that

$$
\dot{F}_{\psi}^{\prime}=\dot{F}_{\psi} \text {, or } \dot{F}_{\psi}^{\prime}=p \dot{F}_{\psi}=F_{\psi} .
$$

Therefore starting from $\mathcal{P}$ or $\mathcal{P}^{\prime}$ the construction (7.4) yields the same $d$ display. Therefore over $\kappa_{F}$ the functor morphism (7.6) factors

$$
\mathcal{M}_{r} \otimes_{O_{F}} \kappa_{F} \longrightarrow \mathcal{M}_{r^{\circ}} \otimes_{O_{F}} \kappa_{F} \longrightarrow \mathcal{M}_{r^{\circ}} \otimes_{O_{F}} \kappa_{F} .
$$

The first morphism is given by Theorem 4.12 and the second morphism is defined by associating to a display of a formal $O_{D^{-}}$-module satisfying $\left(\mathbf{D}_{r^{\circ}}\right)$ the display relative to $O_{F}$. Therefore the last morphism is the identity. Since the first arrow is an isomorphism by Theorem 4.12, we deduce that (7.6) is an isomorphism over $\kappa_{F}$. But since both schemes are flat by Proposition 5.8, it is an isomorphism. 
The only thing we use of Ahsendorf's work is: to each relative display $\mathcal{P}^{\prime}$ there is a formal group $B T\left(\mathcal{P}^{\prime}\right)$. Let $X$ be a formal $O_{D^{-m o d u l e ~ s a t i s f y i n g ~}}$ $\left(\mathbf{D}_{r^{\circ}}\right)$. Let $\mathcal{P}$ be the display of $X$. Let $\mathcal{P}^{\prime}$ be the relative display given by construction (7.5). Then $B T\left(\mathcal{P}^{\prime}\right)$ is canonically isomorphic to $X$.

\section{The Lubin-Tate moduli problem}

In this section we sketch that a modification of our method is applicable to a variant of the Lubin-Tate moduli problem. Let again $F$ be an extension of degree $d$ of $\mathbb{Q}_{p}$. We again fix an embedding $\varphi_{0}: F \longrightarrow \overline{\mathbb{Q}}_{p}$. We also fix an integer $n \geq 2$, and function $r: \Phi \longrightarrow \mathbb{Z}_{>0}$ with the same properties as in (2.1). Let $E$ be the corresponding reflex field.

Let $S$ be an $O_{E^{-}}$scheme. Let $(\mathcal{L}, \iota)$ be a locally free $\mathcal{O}_{S}$-module of finite rank with an action by $O_{F}$. We say that $(\mathcal{L}, \iota)$ is an $O_{F}$-module over $S$.

Let $\tilde{E}$ be the normal closure of $E$. Then each $\varphi \in \Phi$ factors through $\varphi: O_{F} \rightarrow O_{\tilde{E}}$. The induced homomorphism $O_{F} \otimes_{\mathbb{Z}_{p}} O_{\tilde{E}} \rightarrow O_{\tilde{E}}$ defines a map

$$
\mathrm{Nm}_{\varphi}: \mathbb{V}\left(O_{F}\right)_{O_{\tilde{E}}} \longrightarrow \mathbb{A}_{O_{\tilde{E}}}^{1} .
$$

We set $\mathrm{Nm}_{r}=\prod_{\varphi} \mathrm{Nm}_{\varphi}^{r_{\varphi}}$, comp. (3.6). This is a polynomial function defined over $O_{E}$,

$$
\mathrm{Nm}_{r}: \mathbb{V}\left(O_{F}\right)_{O_{E}} \longrightarrow \mathbb{A}_{O_{E}}^{1}
$$

We use the notations $\Psi, a_{\psi}$ from (2.3). For an $O_{E}$-module $\mathcal{L}$ we have a natural decomposition

$$
\mathcal{L}=\bigoplus_{\psi \in \Psi} \mathcal{L}_{\psi} .
$$

We say that $(\mathcal{L}, \iota)$ satisfies the rank condition $\left(\mathbf{R}_{r}^{F}\right)$ if

$$
\operatorname{rank}_{\mathcal{O}_{S}} \mathcal{L}_{\psi_{0}}=a_{\psi_{0}} n+1, \quad \operatorname{rank}_{\mathcal{O}_{S}} \mathcal{L}_{\psi}=a_{\psi} n, \text { for } \psi \neq \psi_{0}
$$

This implies $\operatorname{rank}_{\mathcal{O}_{S}} \mathcal{L}=\sum_{\varphi \in \Phi} r_{\varphi}$. With the notations (2.8) we introduce the Eisenstein conditions $\left(\mathbf{E}_{r}^{F}\right)$ (compare Definition 3.8):

$$
\begin{aligned}
\left(\left(Q_{0} \cdot Q_{A_{\psi_{0}}}\right)(\iota(\pi)) \mid \mathcal{L}_{\psi_{0}}\right) & =0, \\
\bigwedge^{2}\left(Q_{A_{\psi_{0}}}\left(\iota(\pi) \mid \mathcal{L}_{\psi_{0}}\right)\right) & =0, \\
\left(Q_{A_{\psi}}(\iota(\pi)) \mid \mathcal{L}_{\psi}\right) & =0, \text { for } \psi \neq \psi_{0} .
\end{aligned}
$$

We say that $\mathcal{L}$ satisfies $\left(\mathbf{L T}_{r}^{F}\right)$ if $\left(\mathbf{R}_{r}^{F}\right)$ and $\left(\mathbf{E}_{r}^{F}\right)$ are satisfied. 
We say that an $O_{F}$-module $(\mathcal{L}, \iota)$ over an $O_{E}$-scheme $S$ satisfies the Kottwitz condition $\left(\mathbf{K}_{r}^{F}\right)$ if

$$
\mathrm{Nm}_{\mathcal{L}}=\mathrm{Nm}_{r},
$$

an equality of two morphisms from $\mathbb{V}\left(O_{F}\right) \times_{S p e c} \mathbb{Z}_{p} S$ to $\mathbb{A}_{S}^{1}$. The condition is equivalent to the identity of polynomials with coefficients in $\mathcal{O}_{S}$,

$$
\operatorname{char}(\iota(x) \mid \mathcal{L})=\prod_{\varphi}(T-\varphi(x))^{r_{\varphi}}, \forall x \in O_{F},
$$

cf. Remark 3.2.

We will consider $p$-divisible groups $X$ of height $n d$ over $O_{E}$-schemes $S$ with an action $\iota: O_{F} \longrightarrow \operatorname{End}(X)$. We assume that the action on Lie $X$ satisfies the condition $\left(\mathbf{L T}_{r}^{F}\right)$. We will also say that $X$ is of type $r$.

Let us assume that $S=$ Spec $k$ is the spectrum of a perfect field of characteristic $p$. Let $M$ be the Dieudonné module of $X$. We obtain a decomposition

$$
M=\bigoplus_{\psi \in \Psi} M_{\psi},
$$

where $M_{\psi}$ is a free $W(k)$-module of rank $n\left[F: F^{t}\right]$. By the condition $\left(\mathbf{L} \mathbf{T}_{r}^{F}\right)$ we find (compare Proposition 4.6):

$$
\begin{gathered}
\pi^{a_{\psi_{0}}+1} M_{\psi_{0}} \subset V M_{\sigma \psi_{0}} \subset \pi^{a_{\psi_{0}}} M_{\psi_{0}}, \\
V M_{\sigma \psi}=\pi^{a_{\psi}} M_{\psi}, \quad \text { for } \psi \neq \psi_{0} .
\end{gathered}
$$

If we replace $(M, F, V)$ by $\left(M, \oplus \pi^{a_{\psi}} F, \oplus \pi^{-a_{\psi}} V\right)$, we obtain a Dieudonné module of a $p$-divisible group $Y$ of type $r^{\circ}$ for the Drinfeld function $r^{\circ}$. We see that $X$ is a formal $p$-divisible group if and only if $Y$ is. Then $(Y, \iota)$ is of Lubin-Tate type, of [5], i.e., $Y$ is a strict formal $O_{F}$-module of dimension one and $O_{F}$-height $n$. We conclude that over an algebraically closed field $\bar{k}$ there is up to isomorphism a unique formal $p$-divisible group $(X, \iota)$ of type $r$.

In general we say that $(X, \iota)$ over a scheme $S$ is a Lubin-Tate group of type $r$ if it is a formal $p$-divisible group of height $n d$ such that the action of $O_{F}$ on Lie $X$ satisfies $\left(\mathbf{L} \mathbf{T}_{r}^{F}\right)$.

Remark 8.1. In the case $r=r^{\circ}$, the Eisenstein conditions $\left(\mathbf{E}_{r}^{F}\right)$ in $\left(\mathbf{L T}_{r}^{F}\right)$ are redundant. This follows from the flatness of the naive local model in [17], in the case where $d=n, r=1$ in the notation of loc. cit. But it is also easy to see this directly.

From the case $r=r^{\circ}$ we deduce: 
Proposition 8.2. Any Lubin-Tate group $(X, \iota)$ of type $r$ over $\bar{k}$ is isoclinic. Any two Lubin-Tate groups of type $r$ over $\bar{k}$ are isomorphic. Any $O_{F}$-linear quasi-isogeny of height zero between Lubin-Tate groups of type $r$ is an isomorphism.

More generally the proof of Theorem 4.12 shows the following fact.

Proposition 8.3. Let $S$ be a $\bar{k}$-scheme. Then there is an equivalence between the category of Lubin-Tate groups of type $r$ and the category of Lubin-Tate groups of type $r^{\circ}$.

We fix a Lubin-Tate group $\left(\mathbb{X}, \iota_{\mathbb{X}}\right)$ of type $r$ over the residue class field $\bar{k}$ of $\overline{\mathbb{Q}}_{p}$. It is unique up to isomorphism. We may therefore define a functor $\mathcal{M}_{r}^{F}$ on $\operatorname{Nilp}_{O_{\breve{E}}}$. Namely, fixing a framing object $\left(\mathbb{X}, \iota_{\mathbb{X}}\right)$ over $\bar{k}, \mathcal{M}_{r}^{F}$ associates to $S \in \operatorname{Nilp}_{O_{\breve{E}}}$ the set of isomorphism classes of triples $(X, \iota, \rho)$ where $(X, \iota)$ is a Lubin-Tate group of type $r$ over $S$ and $\rho: X \times_{S} \bar{S} \rightarrow \mathbb{X} \times{ }_{\operatorname{Spec}} \bar{k}$ is an $O_{F}$-linear quasi-isogeny of height zero. The formal scheme representing this functor will be denoted by the same symbol.

The main theorem in this section is the following.

Theorem 8.4. The formal scheme $\mathcal{M}_{r}^{F}$ is isomorphic to the formal spectrum of $O_{\breve{E}}\left[\left[t_{1}, \ldots, t_{n-1}\right]\right]$. A Lubin-Tate group of type $r$ satisfies the Kottwitz condition $\left(\mathbf{K}_{r}^{F}\right)$.

We will show now how this follows from the results of previous sections. The Kottwitz condition follows as in the proof of Proposition 4.5. We first note the following consequence of Corollary 8.2.

Corollary 8.5. $\mathcal{M}_{r}^{F}(\bar{k})=\{\mathrm{pt}\}$, hence $\mathcal{M}_{r}^{F}$ is the formal spectrum of a local $O_{\breve{E}}$-algebra $R$ w.r.t. its maximal ideal.

In order to complete the proof of Theorem 8.4, it remains to show that $\mathcal{M}_{r}^{F}$ is formally smooth of relative dimension $n-1$ over $O_{\breve{E}}$. This will follow from the theory of local models.

Let $V$ be a $F$-vector space of dimension $n$ and $\Lambda$ an $O_{F}$-lattice in $V$. Let $\mathbb{M}_{r}^{F}$ be the functor on $\left(\mathrm{Sch} / O_{E}\right)$ such that

$$
\begin{array}{r}
\mathbb{M}_{r}^{F}(S)=\left\{\mathcal{F} \subset \Lambda \otimes_{\mathbb{Z}_{p}} \mathcal{O}_{S} \mid O_{F}\right. \text {-stable direct summand such that } \\
\left.\left(\Lambda \otimes_{\mathbb{Z}_{p}} \mathcal{O}_{S}\right) / \mathcal{F} \text { satisfies }\left(\mathbf{R}_{r}^{F}\right) \text { and }\left(\mathbf{E}_{r}^{F}\right)\right\} .
\end{array}
$$

Then $R$ is isomorphic to the completion of $\mathbb{M}_{r}^{F}$ at a point of $\mathbb{M}_{r}^{F}(\bar{k})$. Hence Theorem 8.4 follows from the next lemma.

Lemma 8.6. $\mathbb{M}_{r}^{F}$ is smooth of relative dimension $n-1$ over $O_{E}$. In fact, $\mathbb{M}_{r}^{F} \otimes_{O_{E}} \bar{k} \simeq \mathbb{P}_{\bar{k}}^{n-1}$. 
Proof. The last statement implies the first. Indeed, then the general and the special fibre are smooth and irreducible of the same dimension $n-1$. The flatness of $\mathbb{M}_{r}^{F}$ follows as in Corollary 5.4. This proves smoothness.

To study the geometric special fiber $\overline{\mathbb{M}^{F}}=\mathbb{M}^{F} \otimes_{O_{E}} \bar{k}$, we follow the method of section 5. Let $W_{0}=\Lambda \otimes_{O_{F^{t}}, \psi_{0}} \bar{k}$. Let $a_{0}=\left|A_{\psi_{0}}\right|$. Then, as in (5.2), we have an identification

$$
\begin{array}{r}
\overline{\mathbb{M}^{F}}(S)=\left\{\mathcal{F}_{0} \subset W_{0} \otimes_{\bar{k}} \mathcal{O}_{S} \mid \pi\right. \text {-stable direct summand, } \\
\left.\left.\left.\operatorname{rank} W_{0, S} / \mathcal{F}_{0}=a_{0} n+1, \text { and } 1^{\prime}\right), 2^{\prime}\right)\right\}
\end{array}
$$

Here we have set $W_{0, S}=W_{0} \otimes_{\bar{k}} \mathcal{O}_{S}$ and $1^{\prime}$ ) and $2^{\prime}$ ) are as in (5.2), i.e.,

$$
\begin{aligned}
& \left.1^{\prime}\right) \quad \pi^{\left(a_{0}+1\right)} \mid\left(W_{0, S} / \mathcal{F}_{0}\right)=0 \\
& \left.2^{\prime}\right) \wedge^{2}\left(\pi^{a_{0}} \mid\left(W_{0, S} / \mathcal{F}_{0}\right)\right)=0 \text {. }
\end{aligned}
$$

Applying Lemma 4.10, we obtain that for $\mathcal{F}_{0} \in \overline{\mathbb{M}^{F}}(S)$, there is a chain of inclusions,

$$
W_{0}^{a_{0}+1} \otimes_{\bar{k}} \mathcal{O}_{S} \subset \mathcal{F}_{0} \subset W_{0}^{a_{0}} \otimes_{\bar{k}} \mathcal{O}_{S},
$$

where $W_{0}^{a_{0}}=\operatorname{Im} \pi^{a_{0}}$, resp. $W_{0}^{a_{0}+1}=\operatorname{Im} \pi^{a_{0}+1}$, is a $\bar{k}$-subspace of dimension $\left(e-a_{0}\right) n$, resp. $\left(e-a_{0}-1\right) n$ of $W_{0}$. Associating now to $\mathcal{F}_{0}$ the submodule $\mathcal{F}_{0} /\left(W_{0}^{a_{0}+1} \otimes_{\bar{k}} \mathcal{O}_{S}\right)$ of $\left(W_{0}^{a_{0}} / W_{0}^{a_{0}+1}\right) \otimes_{\bar{k}} \mathcal{O}_{S}$, we obtain a direct summand of codimension one, i.e., an $S$-valued point of $\mathbb{P}\left(W_{0}^{a_{0}} / W_{0}^{a_{0}+1}\right)$. Since this association is functorial and bijective, the last assertion of Lemma 8.6 follows.

\section{Acknowledgements}

We thank P. Scholze for explaining to us his proof of point (ii) in Theorem 2.8 , and for a critical reading of a first version of this paper. We also thank U. Görtz for helpful discussions.

\section{References}

[1] T. Ahsendorf, $\mathcal{O}$-displays and $\pi$-divisible formal $\mathcal{O}$-modules, Thesis Bielefeld, 2011.

[2] T. Ahsendorf, Ch. Cheng, Th. Zink, $\mathcal{O}$-displays and $\pi$-divisible formal O-modules, J. of Algebra 457 (2016), 129-193.

[3] J.-F. Boutot and H. Carayol, Uniformisation p-adique des courbes de Shimura: les théorèmes de Cerednik et de Drinfeld, in: Courbes modulaires et courbes de Shimura, Astérisque 196-197 (1991), 45-158. 
[4] G. Chenevier, The p-adic analytic space of pseudocharacters of a profinite group and pseudorepresentations over arbitrary rings, in: Automorphic forms and Galois representations. Vol. 1, 221-285, London Math. Soc. Lecture Note Ser., 414, Cambridge Univ. Press, Cambridge, 2014.

[5] V. G. Drinfeld, Coverings of p-adic symmetric regions, Funct. Anal. Appl. 10 (1977), 29-40.

[6] L. Fargues, Cohomologie des espaces de modules de groupes p-divisibles et correspondances de Langlands locales, in: Variétés de Shimura, espaces de Rapoport-Zink et correspondances de Langlands locales, Astérisque 291 (2004), 1-199.

[7] U. Görtz, On the flatness of models of certain Shimura varieties of PEL type, Math. Ann. 321 (2001), 689-727.

[8] U. Görtz, T. Wedhorn, Algebraic geometry I. Schemes with examples and exercises. Advanced Lectures in Mathematics. Vieweg + Teubner, Wiesbaden, 2010. Vieweg Verlag, Wiesbaden, 2010.

[9] Ph. Hartwig, Kottwitz-Rapoport and p-rank strata in the reduction of Shimura varieties of PEL type, Ann. Inst. Fourier (Grenoble) 65 (2015), no. $3,1031-1103$.

[10] R. E. Kottwitz, Isocrystals with additional structure. II, Compositio Math. 109 (1997), no. 3, 255-339.

[11] S. Kudla and M. Rapoport, An alternative description of the Drinfeld p-adic half-plane, Annales de l'Institut Fourier 64 (2014), 1203-1228.

[12] S. Kudla and M. Rapoport, New cases of p-adic uniformization, Astérisque 370 (2015), 207-241.

[13] S. Kudla, M. Rapoport and Th. Zink, On Cherednik uniformization, in preparation.

[14] E. Lau, Displays and formal p-divisible groups, Invent. Math. 171 (2008), no. 3, 617-628.

[15] A. Mihatsch, Relative unitary RZ-spaces and the Arithmetic Fundamental Lemma, arXiv:1611.06520.

[16] J. Nekovar, Level raising and anticyclotomic Selmer groups for Hilbert modular forms of weight two, Canad. J. Math. 64 (2012), 588-668.

[17] G. Pappas and M. Rapoport, Local models in the ramified case. I: The EL-case, J. Alg. Geom. 12 (2003), 107-145. 
[18] G. Pappas, M. Rapoport and B. Smithling, Local models of Shimura varieties, I. Geometry and combinatorics, in: Handbook of moduli (eds. G. Farkas and I. Morrison), vol. III, 135-217, Adv. Lect. in Math. 26, International Press, 2013.

[19] G. Pappas and X. Zhu, Local models of Shimura varieties and a conjecture of Kottwitz, Inventiones math. 194 (2013), 147-254.

[20] M. Rapoport and E. Viehmann, Towards a theory of local Shimura varieties, Münster J. of Math. 7 (2014), 273-326.

[21] M. Rapoport and T. Zink, Period spaces for $p$-divisible groups. Annals of Mathematics Studies, 141, Princeton University Press, Princeton, 1996.

[22] M. Rapoport, B. Smithling and W. Zhang, Arithmetic diagonal cycles on unitary Shimura varieties, in preparation.

[23] K. Ribet, On modular representations of $\mathrm{Gal}(\overline{\mathbb{Q}} / \mathbb{Q})$ arising from modular forms, Invent. math. 100 (1990), 431-476.

[24] P. Scholze and J. Weinstein, Moduli of p-divisible groups, Cambridge J. Math. 1 (2013), 145-237.

[25] Th. Zink, The display of a formal p-divisible group, in: Cohomologies p-adiques et applications arithmétiques, I. Astérisque 278 (2002), $127-$ 248 .

Michael RAPOPORT

MATHEMATISChes Institut DeR UniversitÄt BonN

ENDENiCher Allee 60

53115 BONN

GERMANY

E-mail address: rapoport@math.uni-bonn.de

Thomas Zink

FAKULtät FÜR MATHEMATIK

UNIVERSITÄT BIELEFELD

POSTFACH 100131

33501 BIELEFELD

GERMANY

E-mail address: zink@math.uni-bielefeld.de

Received December 14, 2015 